\title{
Generalized Mean-Reverting 4/2 Factor Model
}

\author{
Yuyang Cheng ${ }^{\dagger}$, Marcos Escobar-Anel ${ }^{*}+\left[\right.$ and Zhenxian Gong ${ }^{\dagger}$ \\ Department of Statistical and Actuarial Sciences, Western University, London, ON N6A 3K7, Canada; \\ yche259@uwo.ca (Y.C.); zgong26@uwo.ca (Z.G.) \\ * Correspondence: marcos.escobar@uwo.ca \\ + These authors contributed equally to this work.
}

Received: 13 September 2019; Accepted: 26 September 2019; Published: 8 October 2019

\begin{abstract}
This paper proposes and investigates a multivariate 4/2 Factor Model. The name 4/2 comes from the superposition of a CIR term and a 3/2-model component. Our model goes multidimensional along the lines of a principal component and factor covariance decomposition. We find conditions for well-defined changes of measure and we also find two key characteristic functions in closed-form, which help with pricing and risk measure calculations. In a numerical example, we demonstrate the significant impact of the newly added $3 / 2$ component (parameter $b$ ) and the common factor $(a)$, both with respect to changes on the implied volatility surface (up to $100 \%$ ) and on two risk measures: value at risk and expected shortfall where an increase of up to $29 \%$ was detected.
\end{abstract}

Keywords: stochastic covariance; $4 / 2$ model; option pricing; risk measures

\section{Introduction}

Continuous-time stochastic covariance models are crucial in capturing many stylized facts in financial data, from heteroscedasticity and fat tails to changing correlations and leverage effects. Early work in this field focused on discrete time models in the form of generalized autoregressive conditional heteroskedasticity (GARCH) models (see Engle 2002). The best-known representatives in continuous time, are the stochastic Wishart family (see Da Fonseca et al. 2007; Gourieroux 2006) and the Ornstein-Uhlenbeck (OU) family (see Muhle-Karbe et al. 2012) of models, as well as general linear-quadratic jump-diffusions (see Cheng and Scaillet 2007). These approaches are more realistic than the classical Black-Scholes lognormal model, but they quickly become intractable as dimensions increase in terms of the number of parameters and simulation paths, commonly known as the "curse of dimensionality". Recent papers (see De Col et al. 2013; Escobar 2018) have presented models built from linear combination of tractable one-dimensional counterparts. These models involve fewer parameters than Wishart- or OU-type approaches, owing to a reduction in dimensionality while providing a closed-form solution to financial problems.

In this paper, we introduce a multivariate mean-reverting stochastic volatility factor model that combines $1 / 2$ (Heston-type, Heston 1993) and $3 / 2$ processes (Platen 1997) for the modeling of volatility. Such underlying volatility processes were coined $4 / 2$ by Grasselli (2017). Our paper takes advantage of the factor structure in asset prices and allows for a mean-reverting structure on the assets thereby aiming at capturing either multivariate commodity behavior or multiple volatility indexes (see Gnoatto et al. 2018 for an alternative multivariate non-mean-reverting generalization based on a pairwise-structure applied to the exchange-rate market). In particular, our setting reduces the dimension of the parametric space which is a way of controlling the "curse of dimensionality" making parameters identifiable and popular estimation methods feasible. Secondly, the presence of independent common and intrinsic factors, each with its own stochastic volatility, enables an elegant separable structure for characteristic functions (c.f.s) and captures several stylized facts, such 
as: stochastic volatility, stochastic correlation among stocks (see Engle 2002), co-movements in the variances (see Diebold and Nerlove 1989), multiple factors in the volatilities (see Heston et al. 2009) and stock correlations (see Da Fonseca et al. 2007). Thirdly, a factor representation is compatible with economical interpretations, where common factors are exogenous variables explaining financial markets, and intrinsic factors relate to companies' intrinsic risks. Lastly, closed-form expressions are available for joint c.f.s; this is useful for derivative pricing and risk management calculations via Fourier transformations, and it makes c.f.-based estimations methods feasible (see Carr and Madan 1999; Caldana and Fusai 2013; Fusai et al. 2018).

The rationale for a $4 / 2$ volatility process rather than a $1 / 2$ or $3 / 2$ model is masterly presented in Grasselli (2017) for a one-dimensional structure. For instance, as observed by the author, the 1/2 process predicts that the implied volatility skew will flatten when the instantaneous volatility increases (crises), while the $3 / 2$ model predicts steepening skews. The empirical violation of the Feller condition in the $1 / 2$ model is also noted, which makes volatility paths stay closer to 0 for a longer period than empirically supported, while the $3 / 2$ model admits extreme paths with spikes in instantaneous volatility. The two processes complement each other as they imply very different dynamics for the evolution of the implied volatility surface. It stands to reason that such a convenient underlying drive for multidimensional structures should be used to improve not only marginal volatility behavior, but also the dependence structure.

We obtain an analytical representation for the c.f. of the vector of asset prices, which is in closed-form for non-mean-reverting nested cases. This type of c.f. is helpful for derivative pricing purposes. We also produce a second conditional c.f. that can be used for exact simulations of the non-mean reverting assets given the terminal volatilities, where the latter can be simulated exactly via chi-squares. We identify a set of conditions that not only produces well-defined changes of measure, but also avoids local martingales; hence, it can be used for risk-neutral pricing purposes.

Our results were applied numerically to parameters inspired by commodity prices ${ }^{1}$. There is a vast literature on commodity modeling (see, for instance, Chiarella et al. 2013; Schwartz 1997, and more recently Schneider and Tavin 2018). In our numerical study, we investigated the impact of the new parameters $(b$, the weight of $3 / 2$ in the overall instantaneous volatility) on the shape of the implied volatility surface and the values of two risk measures: VaR and expected shortfall.

\section{Model Description}

Next, we define the model in a filtered probability space $(\Omega, \mathcal{F}, \mathcal{P}, \mathbb{F})$ where $\mathcal{F}_{0}$ contains all subsets of the $(\mathcal{P}-)$ null sets of $\mathcal{F}$ and $\mathbb{F}$ is right-continuous. We first provide the processes under the historical measure $\mathcal{P}$, then followed by the processes under a (conveniently chosen) risk-neutral measure $\mathcal{Q}$. Suppose that $X(t)=\left(X_{1}(t), \ldots, X_{n}(t)\right)^{\prime}$ is a vector of asset prices with the following $\mathcal{P}$-measure representation:

1 This can also be applied to volatility indexes, such as those reported by the Chicago Board Options Exchange (CBOE), which are clearly a mean-reverting asset class with stochastic volatility. 


$$
\begin{aligned}
\frac{d X_{i}(t)}{X_{i}(t)}= & \left\{L_{i}+c_{i} \sum_{j=1}^{p} a_{i j}^{2}\left(\sqrt{v_{j}(t)}+\frac{b_{j}}{\sqrt{v_{j}(t)}}\right)^{2}-\sum_{j=1}^{n} \beta_{i j} \ln \left(X_{j}(t)\right)+\tilde{c}_{i}\left(\sqrt{\tilde{v}_{i}(t)}+\frac{\tilde{b}_{i}}{\sqrt{\tilde{v}_{i}(t)}}\right)^{2}\right\} d t \\
& +\sum_{j=1}^{p} a_{i j}\left(\sqrt{v_{j}(t)}+\frac{b_{j}}{\sqrt{v_{j}(t)}}\right) d W_{j}(t)+\left(\sqrt{\tilde{v}_{i}(t)}+\frac{\tilde{b}_{i}}{\sqrt{\tilde{v}_{i}(t)}}\right) d \widetilde{W}_{i}(t) \\
d v_{j}(t)= & \alpha_{j}\left(\theta_{j}-v_{j}(t)\right) d t+\xi_{j} \sqrt{v_{j}(t)} d B_{j}^{P}(t), j=1, \ldots, p \\
d \tilde{v}_{i}(t)= & \tilde{\alpha}_{i}\left(\tilde{\theta}_{i}-\tilde{v}_{i}(t)\right) d t+\tilde{\xi}_{i} \sqrt{\tilde{v}_{i}(t)} d \tilde{B}_{i}^{P}(t), i=1, \ldots, n
\end{aligned}
$$

The quadratic variation structure is $\left\langle d B_{j}^{P}(t), d W_{j}(t)\right\rangle=\rho_{j} d t,\left\langle d \widetilde{B}_{i}^{P}(t), d \widetilde{W}_{i}(t)\right\rangle=\widetilde{\rho}_{i} d t$ and zero otherwise. In the language of factor analysis, $a_{i j}$ is the $i j$ th entry of the matrix of factor loadings (A) that captures the correlations among assets. The communalities are represented by $V_{j}(t)=$ $\left(\sqrt{v_{j}(t)}+\frac{b_{j}}{\sqrt{v_{j}(t)}}\right)^{2}$ (in matrix form, $\Lambda_{n x p}=A \operatorname{diag}\left(V^{1 / 2}(t)\right)$ ) and the intrinsic residual variance is $\Psi=\operatorname{diag}(\widetilde{V}(t))$, with $\widetilde{V}_{j}(t)=\left(\sqrt{\tilde{v}_{i}(t)}+\frac{\tilde{b_{i}}}{\sqrt{\widetilde{v}_{i}(t)}}\right)^{2}$. This leads to a factors decomposition of the quadratic variation of asset prices as follows:

$$
\Sigma(t) d t=\left(\Lambda \Lambda^{\prime}+\Psi\right) d t=\left(A \operatorname{diag}(V(t)) A^{\prime}+\operatorname{diag}(\widetilde{V}(t))\right) d t
$$

Whenever necessary, we assume $n=p$ and $A=\left(a_{i j}\right)_{n \times p}$ to be an orthogonal matrix. In this setting, $c_{i}$ and $\tilde{c_{i}}$ represent risk premiums of asset $X_{i}(t)$ associated with the common and intrinsic factors, respectively. $\beta=\left(\beta_{i j}\right)_{n \times n}$ is an invertible matrix, which captures the spillover at the expected return level $X_{i}(t)$ on asset $X_{j}(t)$. In other words, it represents the impact from other assets on the long term average price of the current one.

Based on the quadratic variation relationship defined in this model, if we assume that $B_{j}^{P}, B_{j}^{P}(t)^{\perp}$, $\tilde{B}_{i}^{P}(t), \tilde{B}_{i}^{P}(t)^{\perp}$ are independent Brownian motions with $-1 \leq \rho_{j} \leq 1$ and $-1 \leq \tilde{\rho}_{i} \leq 1$. Then,

$$
\begin{aligned}
& d W_{j}(t)=\rho_{j} d B_{j}^{P}(t)+\sqrt{1-\rho_{j}^{2}} d B_{j}^{P}(t)^{\perp} \\
& d \widetilde{W}_{i}(t)=\tilde{\rho}_{i} d \tilde{B}_{i}^{P}(t)+\sqrt{1-\tilde{\rho}_{i}^{2}} d \tilde{B}_{i}^{P}(t)^{\perp} .
\end{aligned}
$$

$v_{j} j=1, \ldots, n$ and $\tilde{v}_{i} i=1, \ldots, n$ follow standard CIR processes, hence $\alpha_{j}, \theta_{j}$, and $\xi_{j}$ are positive constants satisfying $\alpha_{j} \theta_{j} \geq \frac{\tilde{\xi}_{j}^{2}}{2}$ (the Feller condition). Similarly, $\tilde{\alpha}_{i}, \tilde{\theta}_{i}$, and $\tilde{\xi}_{i}$ are positive constants satisfying $\tilde{\alpha}_{i} \tilde{\theta}_{i} \geq \frac{\tilde{\xi}_{i}^{2}}{2}$. Note that the Feller condition in CIR model guarantees that the process remains positive.

The transformation $Y=\ln X$ would create a multivariate Ornstein-Uhlenbeck process with a $4 / 2$ stochastic factor structure:

$$
\begin{aligned}
d Y_{i}(t)= & \left\{L_{i}+\left(c_{i}-\frac{1}{2}\right) \sum_{j=1}^{n} a_{i j}^{2}\left(\sqrt{v_{j}(t)}+\frac{b_{j}}{\sqrt{v_{j}(t)}}\right)^{2}-\sum_{j=1}^{n} \beta_{i j} Y_{j}(t)+\left(\tilde{c_{i}}-\frac{1}{2}\right)\left(\sqrt{\tilde{v}_{i}(t)}+\frac{\tilde{b_{i}}}{\sqrt{\tilde{v}_{i}(t)}}\right)^{2}\right\} d t \\
& +\sum_{j=1}^{n} a_{i j}\left(\sqrt{v_{j}(t)}+\frac{b_{j}}{\sqrt{v_{j}(t)}}\right) d W_{j}(t)+\left(\sqrt{\tilde{v}_{i}(t)}+\frac{\tilde{b_{i}}}{\sqrt{\tilde{v}_{i}(t)}}\right) d \widetilde{W}_{i}(t)
\end{aligned}
$$


This general model includes a notable particular case, which is a direct generalization of Grasselli (2017) to a factor setting when $\beta_{i j}=0, i, j=1, \ldots, n$; this case is studied in more detail and it is named FG, given its analytical flexibility.

\section{Results}

This section describes viable changes of measure and two key characteristic functions of the targeted multivariate process; one for pricing and the other for simulations. The proofs are presented in Appendix A.

\subsection{Change of Measure}

Here, we explore the topic of creating a risk-neutral measure $\mathcal{Q}$ for pricing purposes. As noted by Grasselli (2017); Platen and Heath (2010) and Baldeaux et al. (2015) among others, a risk-neutral measure may not be supported by data in the presence of a $3 / 2$ model (e.g., $\frac{1}{\sqrt{v(t)}}$ ), as the parametric constraints needed for the discounted asset price process to be a $\mathcal{Q}$ - martingale are violated with real data; hence, we can only produce a strict $\mathcal{Q}$-local martingale (i.e., $\mathcal{Q}$ would be absolute continuous but not equivalent to $\mathcal{P}$ ). In such situation, the standard risk-neutral pricing methodology would fail (biased prices), and we have to turn to the benchmark approach for pricing (see Baldeaux et al. 2015).

The next proposition entertains the following changes of measure with constant $\lambda_{j}, \lambda_{j}^{\perp}, \widetilde{\lambda}_{i}$ and $\tilde{\lambda}_{i}^{\perp}$ (see Escobar and Gong 2019 for other types of changes of measures) then identifies the parametric conditions needed for the existence of a valid risk-neutral measure $\mathcal{Q}$.

$$
\begin{aligned}
d B_{j}^{Q}(t) & =\lambda_{j}\left(\sqrt{v_{j}(t)}+\frac{b_{j}}{\sqrt{v_{j}(t)}}\right) d t+d B_{j}^{P}(t), d \tilde{B}_{i}^{Q}(t)=\widetilde{\lambda}_{i}\left(\sqrt{\tilde{v}_{i}(t)}+\frac{\tilde{b_{i}}}{\sqrt{\tilde{v}_{i}(t)}}\right) d t+d \tilde{B}_{i}^{P}(t) \\
d B_{j}^{Q}(t)^{\perp} & =\lambda_{j}^{\perp}\left(\sqrt{v_{j}(t)}+\frac{b_{j}}{\sqrt{v_{j}(t)}}\right) d t+d B_{j}^{P}(t)^{\perp}, d \tilde{B}_{i}^{Q}(t)^{\perp}=\tilde{\lambda}_{i}^{\perp}\left(\sqrt{\tilde{v}_{i}(t)}+\frac{\tilde{b}_{i}}{\sqrt{\tilde{v}_{i}(t)}}\right) d t+d \tilde{B}_{i}^{P}(t)^{\perp}
\end{aligned}
$$

Proposition 1. The change of measure is well-defined for pricing purposes under the following four conditions:

$$
\begin{gathered}
\widetilde{\xi}_{j}^{2} \leq 2 \alpha_{j} \theta_{j}-2 \widetilde{\xi}_{j} \max \left\{\left|b_{j} \lambda_{j}\right|,\left|\lambda_{j}^{\perp} b_{j}\right|,\left|b_{j} a_{1 j} \rho_{j}\right|, \ldots,\left|b_{j} a_{n j} \rho_{j}\right|\right\} \\
\widetilde{\xi}_{i}^{2} \leq 2 \widetilde{\alpha}_{i} \widetilde{\theta}_{i}-2 \widetilde{\xi}_{i} \max \left\{\left|\widetilde{b}_{i} \widetilde{\lambda}_{i}\right|,\left|\widetilde{\lambda}_{i}^{\perp} \widetilde{b}_{i}\right|,\left|\widetilde{b}_{i} \widetilde{\rho}_{i}\right|\right\} \\
\max \left\{\left|\lambda_{j}\right|,\left|\lambda_{j}^{\perp}\right|\right\}<\frac{\alpha_{j}}{\widetilde{\xi}_{j}} \\
\max \left\{\left|\widetilde{\lambda}_{i}\right|,\left|\widetilde{\lambda}_{i}^{\perp}\right|\right\}<\frac{\widetilde{\alpha}_{i}}{\widetilde{\xi}_{i}}
\end{gathered}
$$

Moreover, if $\beta_{i j}=0$ for $i, j=1, \ldots, n$, then the following must also be satisfied:

$$
L_{i}=r, c_{i}=\sum_{j=1}^{n} a_{i j}\left(\rho_{j} \lambda_{j}+\sqrt{1-\rho_{j}^{2}} \lambda_{j}^{\perp}\right), \widetilde{c}_{i}=\widetilde{\rho}_{i} \widetilde{\lambda}_{i}+\sqrt{1-\widetilde{\rho}_{i}^{2}} \widetilde{\lambda}_{i}^{\perp}
$$

Proof is included in Appendix A. 


\subsection{Characteristic Function}

This section aims at obtaining an analytical representation for the c.f. If $Z(t)=e^{\beta t} Y(t)$ is defined such that $e^{\beta t}$ is a matrix exponential, then $Z_{i}(t)$ is represented as:

$$
\begin{aligned}
d Z_{i}(t)= & \sum_{j=1}^{n}\left(e^{\beta t}\right)_{i j}\left\{L_{i}+\left(c_{j}-\frac{1}{2}\right) \sum_{k=1}^{n} a_{j k}^{2}\left(\sqrt{v_{k}(t)}+\frac{b_{k}}{\sqrt{v_{k}(t)}}\right)^{2}+\left(\tilde{c_{j}}-\frac{1}{2}\right)\left(\sqrt{\tilde{v}_{j}(t)}+\frac{\tilde{b}_{j}}{\sqrt{\tilde{v}_{j}(t)}}\right)^{2}\right\} d t \\
& +\sum_{j=1}^{n}\left(e^{\beta t}\right)_{i j}\left\{\sum_{k=1}^{n} a_{j k}\left(\sqrt{v_{k}(t)}+\frac{b_{k}}{\sqrt{v_{k}(t)}}\right) d W_{k}(t)+\left(\sqrt{\tilde{v}_{j}(t)}+\frac{\tilde{b_{j}}}{\sqrt{\tilde{v}_{j}(t)}}\right) d \widetilde{W}_{j}(t)\right\}
\end{aligned}
$$

For convenience, we use $\left(e^{\beta t}\right)_{i j}$ as the $i j$ component of the matrix $e^{\beta t}$. Note that $Z_{i}(t)$ is no longer a mean-reverting process although it accounts for time dependent coefficients.

Next, we find the conditional c.f. for the increments of $Z$, defined as

$$
\Phi_{Z(t), v(t)}(T, \omega)=E\left[\exp \left\{i \omega^{\prime}(Z(T)-Z(t))\right\} \mid Z(t)=z_{t}, v(t)=v_{t}\right]
$$

Under a risk neutral measure, this c.f. can be used for pricing some financial products, given the integrability conditions (a discussion of the generalized c.f. as per Grasselli 2017 is beyond the scope of this paper.). For convenience, we formulate it as $v(t)=\left(v_{1}(t), \ldots, v_{n}(t), \widetilde{v}_{1}(t), \ldots, \widetilde{v}_{n}(t)\right)$.

Proposition 2. Let $(Z(t))_{t \geq 0}$ evolve according to the model in Equation (6). The c.f. $\Phi_{Z(t), v(t)}$ is then given as follows:

$$
\begin{gathered}
\Phi_{Z(t), v(t)}(T, \omega)=E\left[\exp i \omega^{\prime}(Z(T)-Z(t)) \mid Z(t)=y_{t}, v(t)=v_{t}\right] \\
=\prod_{k=1}^{n} \Phi_{G G}\left(T, 1 ; L_{k}(\omega), h_{k}(\omega), g_{k}(\omega), \kappa_{k}, \theta_{k}, \widetilde{\xi}_{k}, \rho_{k}, b_{k}, c_{k}, v_{k, t}, S_{k, t}^{*}\right) \\
\times \prod_{j=1}^{n} \Phi_{G G}\left(T, 1 ; 0, L_{j}(\omega), h_{j}(\omega), g_{j}(\omega), \widetilde{\kappa}_{j}, \widetilde{\theta}_{j}, \widetilde{\xi}_{j}, \widetilde{\rho}_{j}, \widetilde{b}_{j}, \widetilde{c}_{k}, \widetilde{v}_{j, t}, S_{t}^{* j}\right)
\end{gathered}
$$

where $\Phi_{G G}$ is a one-dimensional generalization of the c.f. from Grasselli (2017) provided in Lemma A1.

Proof is provided in Appendix A. The c.f. above involves single expected values with respect to Brownian motion $B(t)$. In each term, $\Phi_{G G}$ (i.e., the second set of Brownian $W(t)$ ) is eliminated, hence this is a drastic simplification compared to the original $2 n$ dimensional joint expectation.

A particular, fully solvable case is the FG model $\left(\beta_{i j}=0, i, j=1, \ldots, n\right)$.

Corollary 1. Let $(Z(t))_{t \geq 0}$ evolve according to the $F G$ model $\left(\beta_{i j}=0, i, j=1, \ldots, n\right)$. The c.f. $\Phi_{Z(t), v(t)}$ is subsequently presented as follows:

$$
\begin{aligned}
& \Phi_{Z(t), v(t)}(T, \omega)=E\left[\exp i \omega^{\prime}(Z(T)-Z(t)) \mid Z(t)=y_{t}, v(t)=v_{t}\right] \\
& =\prod_{k=1}^{n} \Phi_{G}\left(T, 1 ; L_{k}(\omega), h_{k}(\omega), g_{k}(\omega), \kappa_{k}, \theta_{k}, \widetilde{\xi}_{k}, \rho_{k}, b_{k}, c_{k}, v_{k, t}, S_{k, t}^{*}\right) \\
& \times \prod_{j=1}^{n} \Phi_{G}\left(T, 1 ; 0, L_{j}(\omega), h_{j}(\omega), g_{j}(\omega), \widetilde{\kappa}_{j}, \widetilde{\theta}_{j}, \widetilde{\xi}_{j}, \widetilde{\rho}_{j}, \widetilde{b}_{j}, \widetilde{c}_{k}, \widetilde{v}_{j, t}, S_{t}^{* j}\right)
\end{aligned}
$$

where $\Phi_{G}$ is the one-dimensional c.f provided by Grasselli (2017) in Proposition 3.1 and given in the Appendix B for completeness. 
See Appendix A for proof. Next, we turn to the conditional c.f. of the increments of $Z$ given the terminal value of the CIR processes. This is defined as follows:

$$
\Phi_{Z(t), v(T)}(\tau, \omega)=E\left[\exp \left[\omega^{\prime}(Z(T)-Z(t))\right] \mid Z(t)=z_{t}, v(T)=v_{T}\right]
$$

The above is useful when we need to work with the joint distribution of $(Z(T), v(T))$ given $(Z(t), v(t))$. For such cases, we can try to rely on a convenient simulation scheme combining the distribution of $Z(T)$ given $(Z(t), v(T)$ ) (via Equation (8)) with that of $v(T)$ given $v(t)$, the latter is known to be non-centered chi-squared. In this way, we can avoid usual discretization algorithms such as the Euler-Maruyama or Milstein schemes, which are generally not suitable for the CIR process (due to failure of the Lipschitz condition at 0 ).

In this vein, when working with the non mean-reverting factor model $\left(\beta_{i j}=0, i, j=1, \ldots, n\right)$, we can easily adapt the procedures in Grasselli (2017) to provide an exact simulation scheme for the model given the vector of the independent CIR process at maturity $T$ (i.e., $v(T)$ ). This requires only the c.f. provided next:

Corollary 2. Let $(Z(t))_{t \geq 0}$ evolve according to the $F G$ model $\left(\beta_{i j}=0, i, j=1, \ldots, n\right)$. Then, the c.f. $\Phi_{Z(t), v(T)}$ is then given as follows:

$$
\begin{aligned}
\Phi_{Z(t), v(T)}(T, \omega) & =\prod_{j=1}^{n} \Phi_{G, T}\left(T, \phi ; L, h_{j}, g_{j}, \kappa_{j}, \theta_{j}, \xi_{j}, \rho_{j}, b_{j}, c_{j}, v_{j, T}, S_{j, t}^{*}\right) \\
& \times \prod_{i=1}^{n} \Phi_{G, T}\left(T, 1 ; 0, h_{i}, g_{i}, \widetilde{\kappa}_{i}, \widetilde{\theta}_{i}, \widetilde{\zeta}_{i}, \widetilde{\rho}_{i}, \widetilde{b}_{i}, \widetilde{c}_{i}, \widetilde{v}_{i, T}, S_{t}^{* i}\right)
\end{aligned}
$$

where $\Phi_{G, T}$ is the one-dimensional c.f provided by Grasselli (2017) in Proposition 4.1 and given in the Appendix B for completeness.

Proof of this result is provided in Appendix A. Unsurprisingly, the previous result cannot be extended to the mean-reverting case, due to the absence of closed formulas for the object:

$$
\mathbb{E}\left[\exp \left\{u\left(\int_{t}^{T} B(s) v(s) d s+\int_{t}^{T} C(s) \frac{1}{v(s)} d s+\int_{t}^{T} D(s) \ln (v(s)) d s\right)\right\} \mid v(T)\right]
$$

which is not solvable even when two of the three deterministic functions $B(s), C(s)$ and $D(s)$ are zero.

\section{Discussion: One Common Factor in Two Dimensions}

We assume two assets, i.e., $X_{1}(t)$ and $X_{2}(t)$, with one common volatility component, and one intrinsic factor each. The asset prices thereby follow the system of SDE for $i=1,2$ :

$$
\begin{aligned}
d Y_{i}(t)= & \left(L_{i}-\beta_{i} Y_{i}(t)\right) d t \\
& +\left(\left(c_{i}-\frac{1}{2}\right)\left[a_{i}^{2}\left(\sqrt{v_{1}(t)}+\frac{b_{1}}{\sqrt{v_{1}(t)}}\right)^{2}\right]+\left(\tilde{c_{i}}-\frac{1}{2}\right)\left(\sqrt{\tilde{v}_{i}(t)}+\frac{\tilde{b}_{i}}{\sqrt{\tilde{v}_{i}(t)}}\right)^{2}\right) d t \\
+ & a_{i}\left(\sqrt{v_{1}(t)}+\frac{b_{1}}{\sqrt{v_{1}(t)}}\right) d W_{1}(t)+\left(\sqrt{\widetilde{v}_{i}(t)}+\frac{\widetilde{b}_{i}}{\sqrt{\widetilde{v}_{i}(t)}}\right) d \widetilde{W}_{i}(t) \\
d v_{1}(t)= & \alpha_{1}\left(\theta_{1}-v_{1}(t)\right) d t+\tilde{\zeta}_{1} \sqrt{v_{1}(t)} d B_{1}(t) \\
d \tilde{v}_{i}(t)= & \tilde{\alpha}_{i}\left(\tilde{\theta}_{i}-\tilde{v}_{i}(t)\right) d t+\tilde{\zeta}_{i} \sqrt{\tilde{v}_{i}(t)} d \tilde{B}_{i}(t)
\end{aligned}
$$


with $\left\langle d B_{j}(t), d W_{j}(t)\right\rangle=\rho_{j} d t,\left\langle d \widetilde{B}_{i}(t), d \widetilde{W}_{i}(t)\right\rangle=\widetilde{\rho}_{i} d t$ for $j=1 ; i=1,2$.

The following table (Table 1 ) gives a baseline parameter set for the one-factor, two-dimensional $4 / 2$ factor model used in the subsequent sections. The choice of parameters in Scenario A was made by combining the seminal works of Schwartz (1997) (see Oil and Copper in Tables IV and V) and Heston (1993). Scenario B combines Schwartz (1997) (see Oil and Copper, Tables IV and V) with Heston et al. (2009). In both cases, we assume a simple structure for the market price of risk $\left(c_{1}=c_{2}=\tilde{c_{1}}=\tilde{c_{2}}=0\right)^{2}$.

The $\tilde{\theta}_{i}, i=1,2$ in the table are set to match the long term volatilities as estimated in Schwartz (1997), which are 0.334 (Oil, Table IV) and 0.233 (Copper, Table V):

$$
\begin{aligned}
& \mathbb{E}\left[a_{1}^{2}\left(\sqrt{v_{1}(t)}+\frac{b_{1}}{\sqrt{v_{1}(t)}}\right)^{2}+\left(\sqrt{\tilde{v}_{1}(t)}+\frac{\tilde{b}_{1}}{\sqrt{\tilde{v}_{1}(t)}}\right)^{2}\right] \\
= & a_{1}^{2}\left(\frac{2 \alpha_{1} b_{1}^{2}}{2 \alpha_{1} \theta_{1}-\tilde{\zeta}_{1}^{2}}+2 b_{1}+\theta_{1}\right)+\frac{2 \tilde{\alpha}_{1} \tilde{b}_{1}^{2}}{2 \tilde{\alpha}_{1} \tilde{\theta}_{1}-\tilde{\xi}_{1}^{2}}+2 \tilde{b}_{1}+\tilde{\theta}_{1}=(0.334)^{2}
\end{aligned}
$$

This explains the values of $\tilde{\theta}_{i}$ in the table.

Table 1. Toy parametric values.

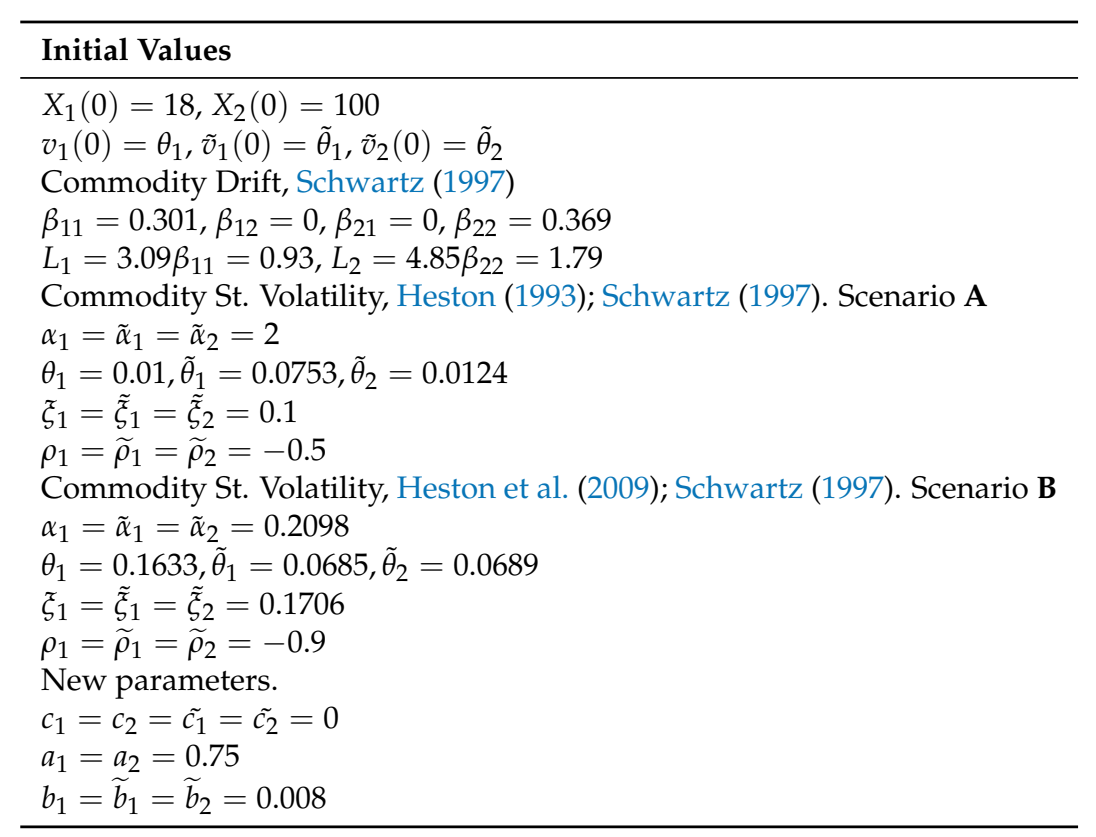

The present section considers two independent cases. First, we study the impact of the parameters $b_{1}, \widetilde{b}_{1}$ and $\widetilde{b}_{2}$ on implied volatility surfaces and on two risk measures for a portfolio of underlyings. We then assess the impact of the commonalities $a_{1}$ and $a_{2}$ on these same targets, i.e., implied volatilities and risk measures. To ensure that the cases lead to reasonable assets behavior, we report the expected return, variance of return for each asset, as well as the correlation between two assets and the leverage effects in Tables 2 and 3 under Scenarios $\mathbf{A}$ and $\mathbf{B}$, respectively.

We simulated 500,000 paths with $d t=0.1$ and considered the following scenarios for $b: b_{1}=0.008$, $\tilde{b}_{1}=\tilde{b}_{2}=0 ; b_{1}=0, \tilde{b}_{1}=\tilde{b}_{2}=0.008 ; b_{1}=\tilde{b}_{1}=\tilde{b}_{2}=0$ and $b_{1}=\tilde{b}_{1}=\tilde{b}_{2}=0.008$.

2 Variations on $c$ will be studied in future research as part of a calibration exercise (see Medvedev and Scaillet 2007 for viable approaches and Escobar and Gschnaidtner 2016 for some pitfalls). 
Table 2. First four moments for scenarios on $3 / 2$ component $(b)$, Scenario A.

\begin{tabular}{lcccc}
\hline & $b_{1}=0.008, \tilde{b}_{1}=\tilde{b}_{2}=0$ & $b_{1}=0, \tilde{b}_{1}=\tilde{b}_{2}=0.008$ & $b_{1}=\tilde{b}_{1}=\tilde{b}_{2}=0$ & $b_{1}=\tilde{b}_{1}=\tilde{b}_{2}=0.008$ \\
\hline $\mathbb{E}\left[\frac{X_{1}(T)-X_{1}(0)}{X_{1}(0)}\right]$ & 0.0494 & 0.0489 & 0.0503 & 0.0480 \\
$\mathbb{E}\left[\frac{X_{2}(T)-X_{2}(0)}{X_{2}(0)}\right]$ & 0.0760 & 0.0754 & 0.0764 & 0.0750 \\
$\mathbb{V}\left[\frac{X_{1}(T)-X_{1}(0)}{X_{1}(0)}\right]$ & 0.0663 & 0.0680 & 0.0618 & 0.0729 \\
$\mathbb{V}\left[\frac{X_{2}(T)-X_{2}(0)}{X_{2}(0)}\right]$ & 0.0367 & 0.0390 & 0.0323 & 0.0445 \\
$\operatorname{Corr}\left(\ln X_{1}(T), \ln X_{2}(T)\right)$ & 0.3194 & 0.0896 & 0.1060 & 0.2799 \\
Corr $\left(\ln X_{1}(T),<\ln X_{1}(T)>\right)$ & -0.4287 & -0.4520 & -0.4443 & -0.4406 \\
$\operatorname{Corr}\left(\ln X_{2}(T),<\ln X_{2}(T)>\right)$ & -0.4148 & -0.4511 & -0.4420 & -0.4280 \\
\hline
\end{tabular}

Table 3. First four moments for scenarios on $3 / 2$ component (b), Scenario B.

\begin{tabular}{lcccc}
\hline & $b_{1}=0.008, \tilde{b}_{1}=\tilde{b}_{2}=0$ & $b_{1}=0, \tilde{b}_{1}=\tilde{b}_{2}=0.008$ & $b_{1}=\tilde{b}_{1}=\tilde{b}_{2}=0$ & $b_{1}=\tilde{b}_{1}=\tilde{b}_{2}=0.008$ \\
\hline $\mathbb{E}\left[\frac{X_{1}(T)-X_{1}(0)}{X_{1}(0)}\right]$ & 0.0514 & 0.0504 & 0.0527 & 0.0499 \\
$\mathbb{E}\left[\frac{X_{2}(T)-X_{2}(0)}{X_{2}(0)}\right]$ & 0.0774 & 0.0775 & 0.0787 & 0.0754 \\
$\mathbb{V}\left[\frac{X_{1}(T)-X_{1}(0)}{X_{1}(0)}\right]$ & 0.0360 & 0.0606 & 0.0247 & 0.1022 \\
$\mathbb{V}\left[\frac{X_{2}(T)-X_{2}(0)}{X_{2}(0)}\right]$ & 0.0359 & 0.1508 & 0.0248 & 0.0723 \\
$\operatorname{Corr}\left(\ln X_{1}(T), \ln X_{2}(T)\right)$ & 0.7533 & 0.0099 & 0.4698 & 0.0156 \\
$\operatorname{Corr}\left(\ln X_{1}(T),<\ln X_{1}(T)>\right)$ & -0.4509 & -0.2031 & -0.5560 & -0.0273 \\
$\operatorname{Corr}\left(\ln X_{2}(T),<\ln X_{2}(T)>\right)$ & -0.4496 & -0.0398 & -0.5517 & -0.2444 \\
\hline
\end{tabular}

Similarly, we considered the following scenarios for $a$ : $a_{1}=a_{2}=0 ; a_{1}=0.75, a_{2}=0 ; a_{1}=0$, $a_{2}=0.75$ and $a_{1}=a_{2}=0.75$. Tables 4 and 5 present key statistics for the returns under Scenarios $\mathbf{A}$ and $\mathbf{B}$, respectively.

Table 4. First four moments for scenarios on commonalities (a), Scenario A.

\begin{tabular}{lcccc}
\hline & $a_{1}=a_{2}=0$ & $a_{1}=0.75, a_{2}=0$ & $a_{1}=0, a_{2}=0.75$ & $a_{1}=a_{2}=0.75$ \\
\hline $\mathbb{E}\left[\frac{X_{1}(T)-X_{1}(0)}{X_{1}(0)}\right]$ & 0.0491 & 0.0492 & 0.0493 & 0.0492 \\
$\mathbb{E}\left[\frac{X_{2}(T)-X_{2}(0)}{X_{2}(0)}\right]$ & 0.0761 & 0.0757 & 0.0746 & 0.0759 \\
$\mathbb{V}\left[\frac{X_{1}(T)-X_{1}(0)}{X_{1}(0)}\right]$ & 0.0658 & 0.0727 & 0.0660 & 0.0732 \\
$\mathbb{V}\left[\frac{X_{2}(T)-X_{2}(0)}{X_{2}(0)}\right]$ & 0.0371 & 0.0370 & 0.0444 & 0.0447 \\
$\operatorname{Corr}\left(\ln X_{1}(T), \ln X_{2}(T)\right)$ & 0.0000 & -0.0011 & 0.0004 & 0.2841 \\
$\operatorname{Corr}\left(\ln X_{1}(T),<\ln X_{1}(T)>\right)$ & -0.4523 & -0.4400 & -0.4529 & -0.4430 \\
$\operatorname{Corr}\left(\ln X_{2}(T),<\ln X_{2}(T)>\right)$ & -0.4507 & -0.4521 & -0.4285 & -0.4279 \\
\hline
\end{tabular}

Table 5. First four moments for scenarios on commonalities (a), Scenario B.

\begin{tabular}{lcccc}
\hline & $a_{1}=a_{2}=0$ & $a_{1}=0.75, a_{2}=0$ & $a_{1}=0, a_{2}=0.75$ & $a_{1}=a_{2}=0.75$ \\
\hline $\mathbb{E}\left[\frac{X_{1}(T)-X_{1}(0)}{X_{1}(0)}\right]$ & 0.0514 & 0.0496 & 0.0512 & 0.0500 \\
$\mathbb{E}\left[\frac{X_{2}(T)-X_{2}(0)}{X_{2}(0)}\right]$ & 0.0768 & 0.0772 & 0.0756 & 0.0752 \\
$\mathbb{V}\left[\frac{X_{1}(T)-X_{1}(0)}{X_{1}(0)}\right]$ & 0.0420 & 0.0733 & 0.0420 & 0.0857 \\
$\mathbb{V}\left[\frac{X_{2}(T)-X_{2}(0)}{X_{2}(0)}\right]$ & 0.0507 & 0.0564 & 0.0746 & 0.0719 \\
$\operatorname{Corr}\left(\ln X_{1}(T), \ln X_{2}(T)\right)$ & 0.0004 & -0.0000 & -0.0005 & 0.0039 \\
$\operatorname{Corr}\left(\ln X_{1}(T),<\ln X_{1}(T)>\right)$ & -0.1126 & -0.0047 & -0.3203 & -0.2634 \\
$\operatorname{Corr}\left(\ln X_{2}(T),<\ln X_{2}(T)>\right)$ & -0.1600 & -0.2544 & -0.0164 & -0.0111 \\
\hline
\end{tabular}

\subsection{Pricing Option}

The section prices European call option on the asset $X_{1}$ based on our $4 / 2$ generalized factor model. It explores the implied volatility surface in a three-dimensional plot with strike prices as the $x$-axis, time to maturity as the y-axis, and corresponding implied volatility as z-axis. We take the strike prices 
$K$ to be $15,16.4,17.8,19.2,20.6$, and 22 and the expiry dates $T$ are $0.2,0.36,0.52,0.68,0.84$ and 1.0 . By choosing these strike prices, we account for the in-the-money, at-the-money, and out-of-the-money options, given the initial asset price 18. Subsequently, for each strike price and expiry date, we can obtain a simulated call price as follows

$$
c(T, K)=e^{-r T} \mathbb{E}^{Q}\left[\left(X_{1}(T)-K\right)^{+}\right],
$$

where $X_{1}(T)$ is approximated using the Euler method.

We extract the implied volatility by matching the Black-Scholes option price formula with simulated call prices and solve for the volatility parameter. Hence, we can treat the dynamics of $Y(t)$ as an $\mathrm{O}-\mathrm{U}$ process such that:

$$
d Y(t)=\left(L_{1}-0.5 \sigma^{2}-\beta Y(t)\right) d t+\sigma d W^{*}(t) .
$$

Next, we consider the two cases described above. The first one studies the impact of $b$, which represents the size of the $3 / 2$ component on the covariance; and the second examines the impact of $a$, the size of the commonality.

In the case of $b$, we first extract the implied volatility surface by matching the standard BS formula for changes on $b$ and $\tilde{b}$ respectively (see Figures 1 and 2).

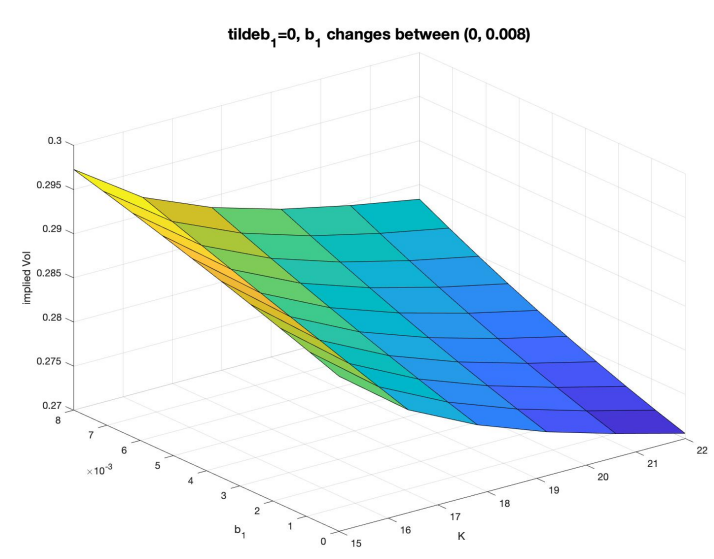

(a) $\tilde{b}_{1}=0, b_{1}$ between $(0,0.008)$

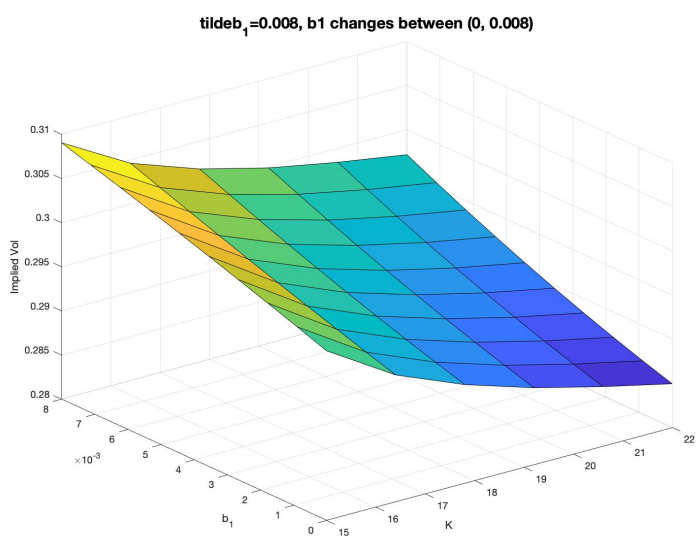

(b) $\tilde{b}_{1}=0.008, b_{1}$ between $(0,0.008)$

Figure 1. Impact of $b_{1}$ (common factor, $3 / 2$ component) on implied volatility, Scenario A.

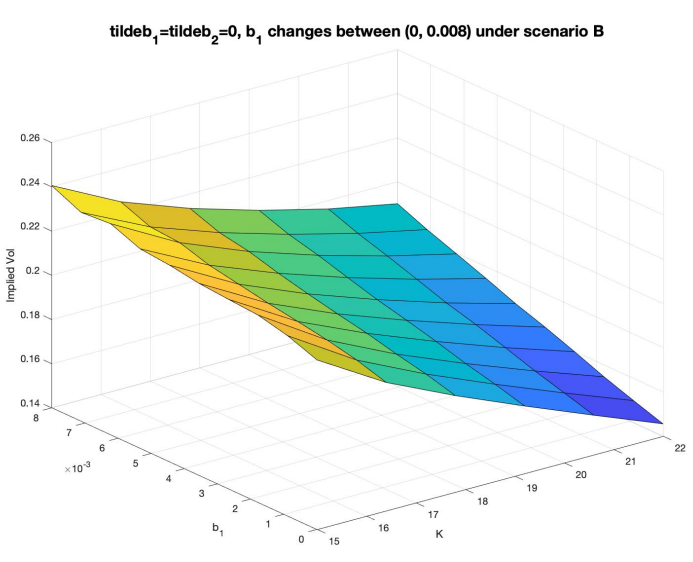

(a) $\tilde{b}_{1}=0, b_{1}$ between $(0,0.008)$

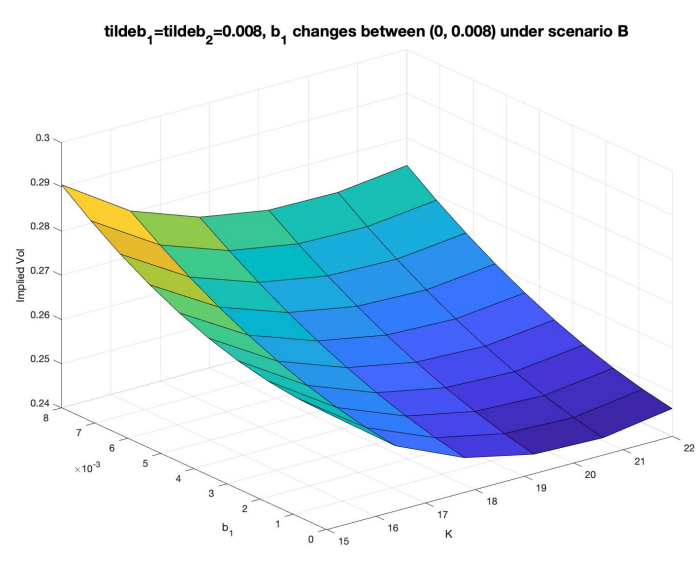

(b) $\tilde{b}_{1}=0.008, b_{1}$ between $(0,0.008)$

Figure 2. Impact of $b_{1}$ (common factor, $3 / 2$ component) on implied volatility, Scenario B. 
For Scenario A, Figure $1 \mathrm{a}, \mathrm{b}$ illustrates that even small changes in $\left(b_{1}\right)$ the common factor $3 / 2$ component (from 0 to 0.008 ) can lead to a $7 \%$ difference in implied volatility (from 0.275 to 0.295 , or 0.285 to 0.305$)$. The joint effect of the common and intrinsic $3 / 2$ components $\left(b_{1}\right.$ and $\left.\tilde{b}_{1}\right)$ can be obtained by combining those two figures leading to a $11 \%$ change (from 0.275 to 0.305 ) in the presence of relatively small values of $b$.

For Scenario B, we observe that the impact of intrinsic factor on volatility surface is more significant than in Scenario A through a comparison of Figure 2a,b. The effect of $b_{1}$ on implied volatility increase by approximately $31 \%(0.145$ to 0.19$)$, as shown in Figure 2a, when only the common factor is present. In Figure $2 b$, we observe a volatility "smile" with the difference of approximately $12.2 \%(0.245$ to 0.275$)$. The joint effect of the common and intrinsic $3 / 2$ components in this case is $100 \%$ (0.145 to 0.29$)$.

Figures 1 and 2 jointly demonstrate that, given different underlying process for common and intrinsic factors, the impact of the $3 / 2$ component can be crucial.

Next, we study $a$, the weight of the common factor (commonality). We again extract the implied volatility surface from matching the standard BS formula for changes on $a$.

Figure $3 \mathrm{a}, \mathrm{b}$ displays the significant increase in implied volatility due to the commonality of the asset with the market $\left(a_{1}\right)$. The change in implied volatility can increase up to $12.5 \%$ (from 0.28 to 0.315 ) in Scenario $\mathbf{A}$ and up to $30 \%$ (from 0.22 to 0.32 ) in Scenario $\mathbf{B}$.

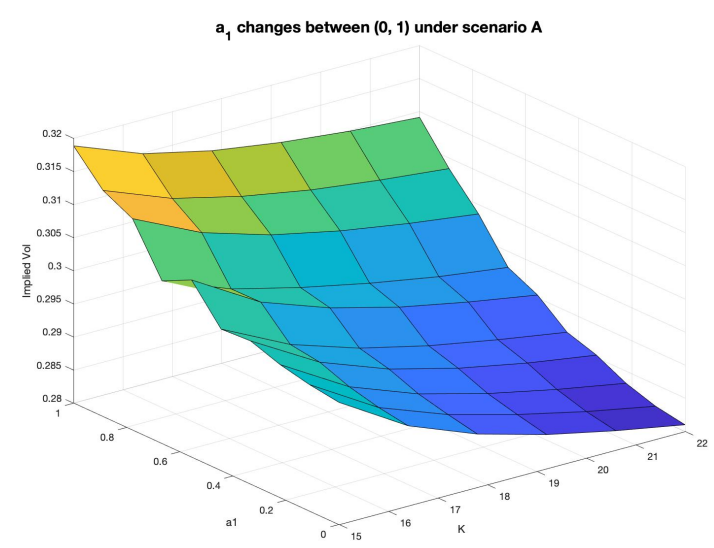

(a) $a_{1}$ between $(0,1)$. Scenario $\mathbf{A}$

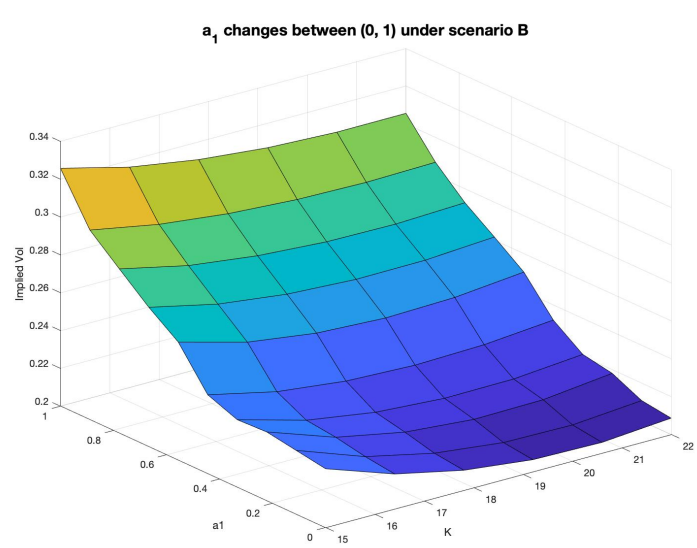

(b) $a_{1}$ between $(0,1)$. Scenario $\mathbf{B}$

Figure 3. Impact of commonality $\left(a_{1}\right)$ on implied volatility.

\subsection{Risk Measures}

This section examines the impact of $b$ and $a$ on important risk measures, in particular the value at risk (VaR) and the expected shortfall (ES). For clarity and calculation purposes, these measures are defined as follows:

$$
\begin{aligned}
\alpha & =P\left(X(T) \leq-V a R_{\alpha}\right) \\
E S & =-\frac{1}{\alpha} \int_{0}^{\alpha} V a R_{\gamma} d \gamma
\end{aligned}
$$

where $X(T)=\omega_{1}\left(X_{1}(T)-X_{1}(0)\right)+\omega_{2}\left(X_{2}(T)-X_{2}(0)\right)$ is the profit and loss portfolio with equal weights $\left(w_{1}=w_{2}=1 / 2\right)$ (see DeMiguel et al. 2007 for a rationale and support of this simple strategy). We let $\alpha$ vary from 0.001 to 0.2 with a discretization size of 200 .

We first study the impact of $b_{1}, \tilde{b}_{1}$ and $\tilde{b}_{2}$ on VaR and ES for a fixed value of $\alpha=0.01$. Figure $4 \mathrm{a}, \mathrm{b}$ illustrates a substantial increase in VaR, from 16 (when all $b$ values are set to zero) to 19.5 (all $b$ set to $0.008)$, which is a $21 \%$ increase $(\alpha=0.01)$ due to the presence of $b$. In other words, an investor would have to set aside $21 \%$ more capital in the presence of $3 / 2$ components. Similarly, ES increases from 
-21 in the presence of $3 / 2$ components to -18.5 in their absence, which constitutes a $12 \%$ increase in the average VaR.

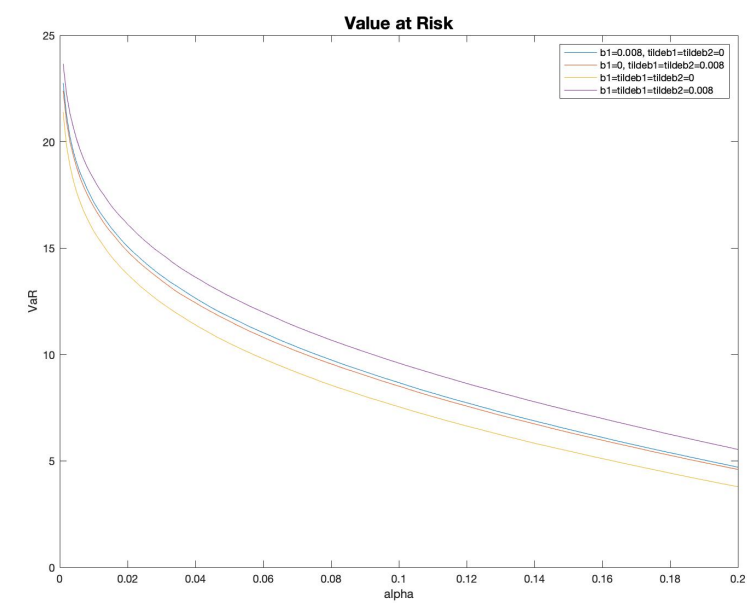

(a) Value at Risk vs. $\alpha$, various b

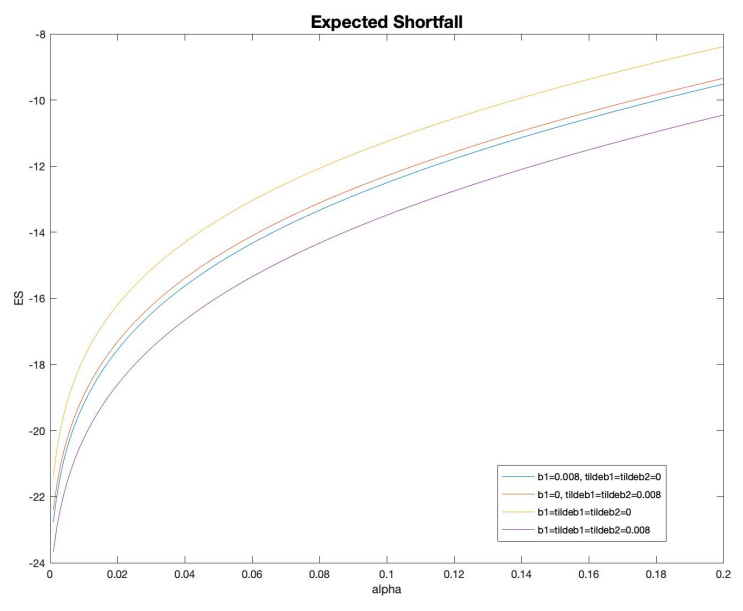

(b) Expected Shortfall vs. $\alpha$, various b

Figure 4. Impact of $3 / 2$ components $(b)$ on Risk measures, Scenario A.

Figure $5 \mathrm{a}, \mathrm{b}$ also displays a substantial increase in VaR, from 17.5 (all $b$ set to zero) to 22.5 ( $b$ set to 0.008$)$, which represents a $28.6 \%$ increase $(\alpha=0.01)$ due to $b$. This means $28.6 \%$ more capital is required in the presence of $3 / 2$ components. Similarly, the ES increases from -27 with $3 / 2$ components to -19 without them, representing a $29.6 \%$ increase in the average VaR.

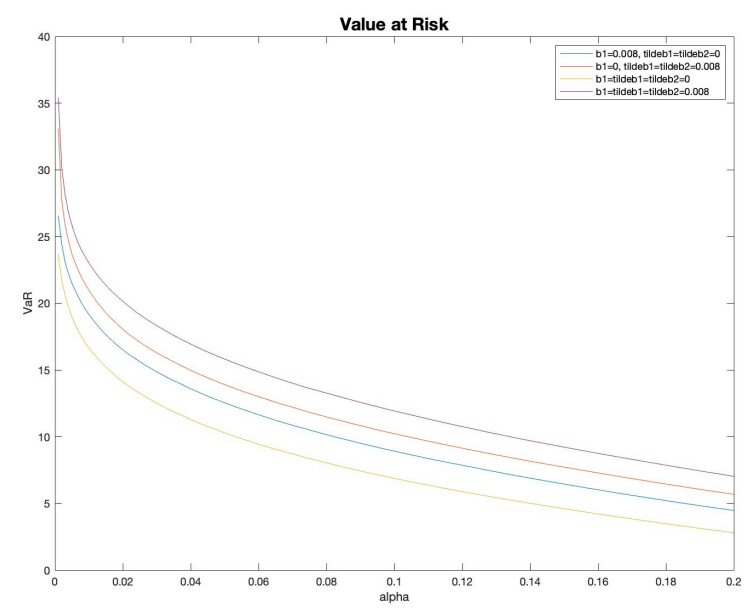

(a) Value at Risk vs. $\alpha$, various b

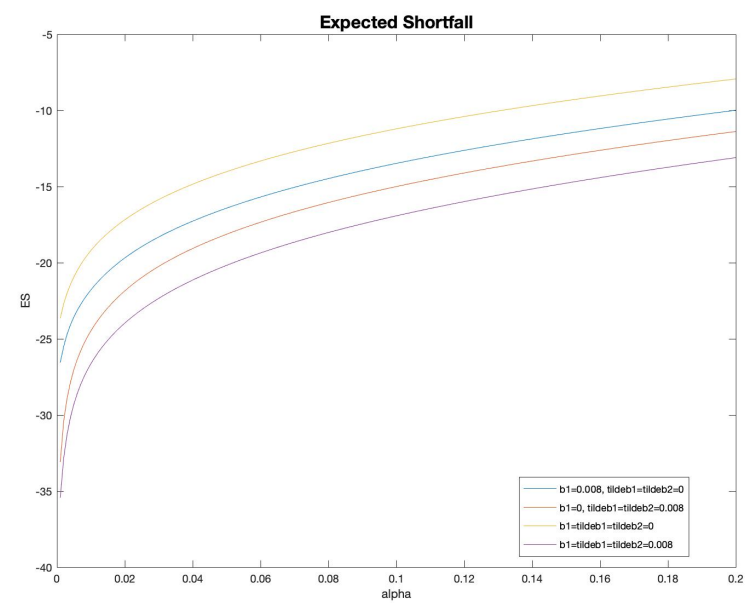

(b) Expected Shortfall vs. $\alpha$, various b

Figure 5. Impact of $3 / 2$ components $(b)$ on Risk measures, Scenario B.

A similar analysis was performed with respect to the commonality $a$, in the presence of stochastic volatility (in the common factor) versus constant volatility; in other words, we assessed the impact of $a$ per se and that of stochastic correlation produced by the $4 / 2$ model. Figure $6 a$ demonstrates an increase in VaR, from 16 to 18.5 , a $15.5 \%$ increase $(\alpha=0.01)$. Figure $6 \mathrm{~b}$ shows that the VaR jumps from 17 to 23 , a $35 \%$ growth due to the increase in $a$. 


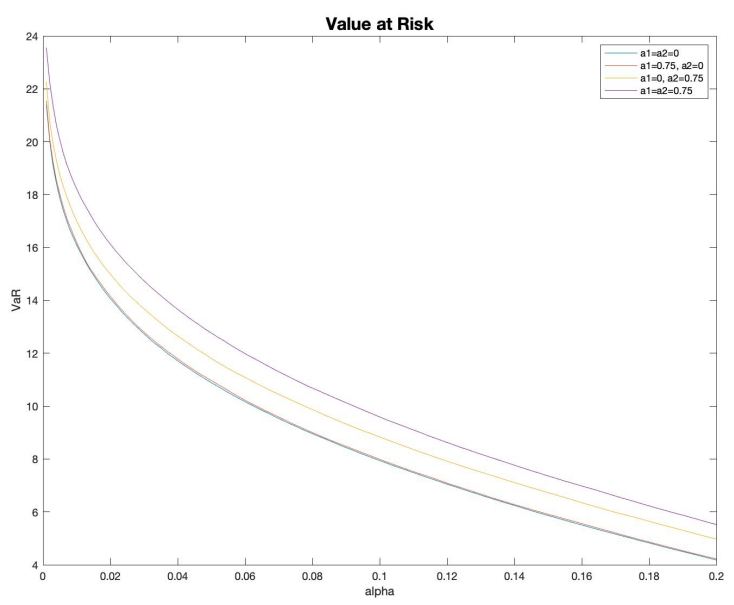

(a) Scenario A: Value at Risk vs. $\alpha$ for different commonality (a) values.

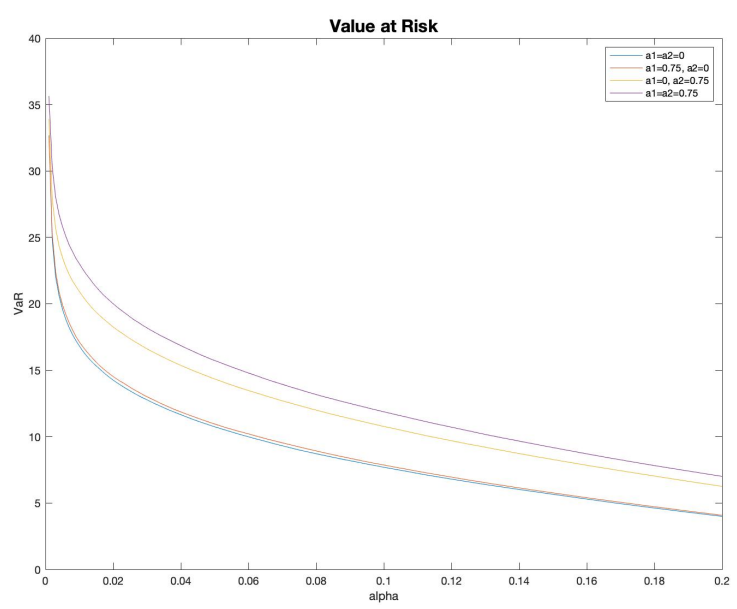

(b) Scenario B: Value at Risk vs. $\alpha$ for different commonality (a) values.

Figure 6. Impact of commonality ( $a$ ) on Value at Risk under Scenario A and Scenario B.

\section{Conclusions}

The generalized men-reverting $4 / 2$ factor model is proposed and studied in this paper. We provide analytical expressions for key characteristic functions and conditions for well-defined changes of measures. We also explore the impact of $b$, i.e., the $3 / 2$ component of the model in the volatility process, and $a$, the commonalities in the absence and presence of stochastic volatility on the common factor. These impacts were measured with respect to implied volatility surfaces and two important risk measures. The results demonstrate that even small values of the $3 / 2$ component $(b)$ can lead to a $100 \%$ change in the implied volatility surface, as well as up to $28 \%$ and $29 \%$ increases in the VaR and ES measures, respectively.

Author Contributions: All authors contributed equally.

Funding: This research received no external funding.

Conflicts of Interest: The authors declare no conflict of interest.

\section{Appendix A. Proofs}

Proof. Proof of Proposition 1.

The first step is to ensure the change of measure is well-defined and for this we use Novikov's condition, i.e., generically

$$
\mathbb{E}\left[\exp \left(\frac{1}{2} \int_{0}^{T} \lambda^{2}\left(\sqrt{v(t)}+\frac{b}{\sqrt{v(t)}}\right)^{2} d s\right)\right]=e^{\lambda^{2} b T} \mathbb{E}\left[\exp \left(\frac{\lambda^{2}}{2} \int_{0}^{T} v(s) d s+\frac{\lambda^{2} b^{2}}{2} \int_{0}^{T} \frac{1}{v(s)} d s\right)\right]<\infty .
$$

From Grasselli, in order for this expectation to exist, we need two conditions:

$$
-\frac{\lambda^{2}}{2}>-\frac{\alpha^{2}}{2 \xi^{2}} \Longrightarrow|\lambda|<\frac{\alpha}{\xi}
$$

and

$$
-\frac{\lambda^{2} b^{2}}{2} \geq-\frac{\left(2 \alpha \theta-\xi^{2}\right)^{2}}{8 \xi^{2}} \Longrightarrow|\lambda| \leq \frac{2 \alpha \theta-\xi^{2}}{2|b| \xi} \Longrightarrow \xi^{2} \leq 2 \alpha \theta-2|\lambda||b| \xi
$$

The latter condition in Equation (A2) implies, in particular, that our volatility processes satisfy Feller's condition under $\mathcal{P}$ and $\mathcal{Q}$; in other words, it ensures all our CIR processes stay away from zero under both measures. 
Applying Equation (A2) to our setting leads to $(i, j=1, \ldots, n)$ :

$$
\begin{aligned}
& \widetilde{\xi}_{j}^{2} \leq 2 \alpha_{j} \theta_{j}-2 \widetilde{\xi}_{j} \max \left\{\left|\lambda_{j} b_{j}\right|,\left|\lambda_{j}^{\perp} b_{j}\right|\right\} \\
& \widetilde{\xi}_{i}^{2} \leq 2 \widetilde{\alpha}_{i} \widetilde{\theta}_{i}-2 \widetilde{\xi}_{i} \max \left\{\left|\widetilde{\lambda}_{i} \widetilde{b}_{i}\right|,\left|\widetilde{\lambda}_{i}^{\perp} \widetilde{b}_{i}\right|\right\}
\end{aligned}
$$

Now, we apply Equation (A1) producing two extra set of conditions $(i, j=1, \ldots, n)$ :

$$
\begin{aligned}
& \max \left\{\left|\lambda_{j}\right|,\left|\lambda_{j}^{\perp}\right|\right\}<\frac{\alpha_{j}}{\widetilde{\xi}_{j}} \\
& \max \left\{\left|\widetilde{\lambda}_{i}\right|,\left|\widetilde{\lambda}_{i}^{\perp}\right|\right\}<\frac{\widetilde{\alpha}_{i}}{\widetilde{\xi}_{i}}
\end{aligned}
$$

The second step applies to the case $\beta_{i j}=0$ for $i, j=1, \ldots, n$ and it is to ensure the drift of the asset price equal the short rate:

$$
L_{i}=r, c_{i}=\sum_{j=1}^{n} a_{i j}\left(\rho_{j} \lambda_{j}+\sqrt{1-\rho_{j}^{2}} \lambda_{j}^{\perp}\right), \widetilde{c}_{i}=\widetilde{\rho}_{i} \widetilde{\lambda}_{i}+\sqrt{1-\widetilde{\rho}_{i}^{2}} \widetilde{\lambda}_{i}^{\perp}
$$

For the most general case $\left(\beta_{i j} \neq 0\right.$ for some $i$ or $\left.j\right)$, the second step should be adapted to any particular prescribed drift structure under the $\mathcal{Q}$-measure.

The third step is to ensure the drift-less asset price process is a true $\mathcal{Q}$-martingale and not just a local $\mathcal{Q}$-martingale:

$$
\frac{d X_{i}(t)}{X_{i}(t)}=(.) d t+\sum_{j=1}^{n} a_{i j}\left(\sqrt{v_{j}(t)}+\frac{b_{j}}{\sqrt{v_{j}(t)}}\right) d W_{j}^{Q}(t)+\left(\sqrt{\tilde{v}_{i}(t)}+\frac{\tilde{b}_{i}}{\sqrt{\tilde{v}_{i}(t)}}\right) d \widetilde{W}_{i}^{Q}(t)
$$

Here, we test the martingale property using the Feller nonexplosion test for volatilities, hence considering the following $n^{2}+n$ changes of Brownian motion for the volatility processes and checking the processes do not reach zero under the various measures:

$$
d B_{i j}^{Q}(t)=a_{i j} \rho_{j}\left(\sqrt{v_{j}(t)}+\frac{b_{j}}{\sqrt{v_{j}(t)}}\right) d t+d B_{j}^{P}(t), d \tilde{B}_{i}^{Q}(t)=\widetilde{\rho}_{i}\left(\sqrt{\tilde{v}_{i}(t)}+\frac{\tilde{b}_{i}}{\sqrt{\tilde{v}_{i}(t)}}\right) d t+d \tilde{B}_{i}^{P}(t)
$$

This leads to the following conditions:

$$
\begin{aligned}
& \xi_{j}^{2} \leq 2 \alpha_{j} \theta_{j}-2\left|a_{i j} \rho_{j} b_{j}\right| \xi_{j}, i, j=1, \ldots, n \\
& \widetilde{\xi}_{i}^{2} \leq 2 \widetilde{\alpha}_{i} \widetilde{\theta}_{i}-2\left|\widetilde{\lambda}_{i} \widetilde{\rho}_{i} \widetilde{b}_{i}\right| \widetilde{\xi}_{i}, i=1, \ldots, n
\end{aligned}
$$

We can combine the first and third steps in Equations (A3), (A7), (A4) and (2) into the final conditions. 
Proof. Proof of Proposition 2.

We start by defining new processes $d S_{i k, t}$ and $d S_{t}^{i j}$ with $i, j, k=1,2, \ldots, n$ and $t \geq 0$.

$$
\begin{aligned}
d Z_{i}(t) & =\sum_{j=1}^{n}\left(e^{\beta t}\right)_{i j}\left[\left(\widetilde{c}_{j}-\frac{1}{2}\right)\left(\sqrt{\tilde{v}_{j}(t)}+\frac{\tilde{b}_{j}}{\sqrt{\tilde{v}_{j}(t)}}\right) d t+\left(\sqrt{\tilde{v}_{j}(t)}+\frac{\tilde{b}_{j}}{\sqrt{\tilde{v}_{j}(t)}}\right) d \widetilde{W}_{j}\right] \\
& +\sum_{k=1}^{n}\left[\left(\sum_{j=1}^{n}\left(e^{\beta t}\right)_{i j} \frac{L_{j}}{n}\right) d t+\left(\sum_{j=1}^{n}\left(e^{\beta t}\right)_{i j}\left(c_{k}-\frac{1}{2}\right) a_{j k}^{2}\right)\left(\sqrt{v_{k}(t)}+\frac{b_{k}}{\sqrt{v_{k}(t)}}\right)^{2} d t\right] \\
& +\sum_{k=1}^{n}\left(\sum_{j=1}^{n}\left(e^{\beta t}\right)_{i j} a_{j k}\right)\left(\sqrt{v_{k}(t)}+\frac{b_{k}}{\sqrt{v_{k}(t)}}\right) d W_{k} \\
& =\sum_{j=1}^{n} d S_{t}^{i j}+\sum_{k=1}^{n} d S_{i k, t}
\end{aligned}
$$

By the dependence structure implied by the model, it follows that all $S$ are independent for a fix $i$, hence we can transform the characteristic function using the processes $S$ as follows:

$$
\Phi_{Z(t), v(t)}(T, \omega)=\prod_{k=1}^{n} E\left[\exp \left\{i \omega^{\prime}\left(S_{\cdot k, T}-S_{\cdot k, t}\right)\right\} \mid S_{t}, v(t)\right] \prod_{j=1}^{n} E\left[\exp \left\{i \omega_{i}\left(S_{T}^{\cdot j}-S_{t}^{j}\right)\right\} \mid S_{t}, v(t)\right]
$$

For each factor $j=1,2, \ldots, n$ we define $S_{k, t}^{*}=\omega^{\prime} S_{\cdot k, t}=\sum_{i=1}^{n} \omega_{i} S_{i k, t}$; the dynamics of $S_{k, t}^{*}$ can be expressed as

$$
\begin{aligned}
& d S_{k, t}^{*}=\omega^{\prime} d S_{\cdot k, t} \\
& =\left[L(\omega, t)+h_{k}(\omega, t)\left(\sqrt{v_{k}(t)}+\frac{b_{k}}{\sqrt{v_{k}(t)}}\right)^{2}\right] d t+g_{k}(\omega, t)\left(\sqrt{v_{k}(t)}+\frac{b_{k}}{\sqrt{v_{k}(t)}}\right) d W_{k, t}
\end{aligned}
$$

where $h_{k}(\omega, t)=\sum_{j=1}^{n}\left(c_{k}-\frac{1}{2}\right) a_{j k}^{2} f_{j}(\omega, t), L(\omega, t)=\sum_{j=1}^{n} \frac{L_{j}}{n} f_{j}(\omega, t)$ and $g_{k}(\omega, t)=\sum_{j=1}^{n} a_{j k} f_{j}(\omega, t)$ and $f_{j}(\omega, t)=\sum_{i=1}^{n} \omega_{i}\left(e^{\beta t}\right)_{i j}$. These three functions are deterministic, linear combinations of $f_{j}(\omega, t)$.

Next, we find the characteristic function for the increments of $S_{k, t}^{*}$ :

$E\left[\exp \left\{i \phi\left(S_{k, T}^{*}-S_{k, t}^{*}\right)\right\} \mid S_{k, t}^{*}, v_{k}(t)=v_{k, t}\right]=\Phi_{G G}\left(T, \phi ; L(\omega), h_{k}(\omega), g_{k}(\omega), \kappa_{k}, \theta_{k}, \xi_{k}, \rho_{k}, b_{k}, c_{k}, v_{k, t}, S_{k, t}^{*}\right)$

The generic function $\Phi_{G G}$ is provided in Lemma A1.

It follows similarly for idiosyncratic factors:

$$
\begin{aligned}
d S_{t}^{* j} & =\omega^{\prime} d S_{t}^{j} \\
& =\left(\sum_{i=1}^{n} \omega_{i}\left(\sum_{j=1}^{n}\left(e^{\beta t}\right)_{i j}\left(\widetilde{c}_{j}-\frac{1}{2}\right)\right)\right)\left(\sqrt{\tilde{v}_{j}(t)}+\frac{\tilde{b}_{j}}{\sqrt{\tilde{v}_{j}(t)}}\right) d t \\
& +\left(\sum_{i=1}^{n} \omega_{i} \sum_{j=1}^{n}\left(e^{\beta t}\right)_{i j}\right)\left(\sqrt{\tilde{v}_{j}(t)}+\frac{\tilde{b_{j}}}{\sqrt{\tilde{v}_{j}(t)}}\right) d \widetilde{W}_{j, t} \\
& =h_{j}(\omega, t)\left(\sqrt{\tilde{v}_{j}(t)}+\frac{\tilde{b}_{j}}{\sqrt{\tilde{v}_{j}(t)}}\right)^{2} d t+g_{j}(\omega, t)\left(\sqrt{\tilde{v}_{j}(t)}+\frac{\tilde{b}_{j}}{\sqrt{\tilde{v}_{j}(t)}}\right) d \widetilde{W}_{j, t}
\end{aligned}
$$

where $h_{j}(\omega, t)=\sum_{i=1}^{n} \omega_{i}\left(\sum_{j=1}^{n}\left(e^{\beta t}\right)_{i j}\left(\widetilde{c}_{j}-\frac{1}{2}\right)\right)$ and $g_{j}(\omega, t)=\sum_{i=1}^{n} \omega_{i} \sum_{j=1}^{n}\left(e^{\beta t}\right)_{i j}$. 
Combining all pieces together, we obtain:

$$
\begin{aligned}
& \Phi_{Z(t), v(t)}(T, \omega)=\prod_{k=1}^{n} E\left[\exp \left\{i \omega^{\prime}\left(S_{\cdot k, T}-S_{\cdot k, t}\right)\right\} \mid S_{t}, v(t)\right] \prod_{j=1}^{n} E\left[\exp \left\{i \omega^{\prime}\left(S_{T}^{\cdot j}-S_{t}^{\cdot j}\right)\right\} \mid S_{t}, v(t)\right] \\
& =\prod_{k=1}^{n} \Phi_{G G}\left(T, 1 ; L_{k}(\omega), h_{k}(\omega), g_{k}(\omega), \kappa_{k}, \theta_{k}, \widetilde{\zeta}_{k}, \rho_{k}, b_{k}, c_{k}, v_{k, t}, S_{k, t}^{*}\right) \\
& \times \prod_{j=1}^{n} \Phi_{G G}\left(T, 1 ; 0, h_{j}(\omega), g_{j}(\omega), \widetilde{\kappa}_{j}, \widetilde{\theta}_{j}, \widetilde{\zeta}_{j}, \widetilde{\rho}_{j}, \widetilde{b}_{j}, \widetilde{c}_{j}, \widetilde{v}_{j, t}, S_{t}^{* j}\right)
\end{aligned}
$$

Lemma A1. Let the generic process be:

$$
\begin{aligned}
& d Z(t)=\left[L(t)+h(t)\left(\sqrt{v(t)}+\frac{b}{\sqrt{v(t)}}\right)^{2}\right] d t+g(t)\left(\sqrt{v(t)}+\frac{b}{\sqrt{v(t)}}\right) d W_{t} \\
& d v(t)=\alpha(\theta-v(t)) d t+\xi \sqrt{v(t)} d B(t) \\
& \langle d B(t), d W(t)\rangle=\rho d t
\end{aligned}
$$

with $g(t)$ differentiable, then

$$
\begin{aligned}
& \Phi_{G G}\left(T, \phi ; L, h, g, \kappa, \theta, \xi, \rho, b, c, v_{t}, Z_{t}\right)=\exp \left\{u \int_{t}^{T} A(s) d s\right\} v(t)^{u \frac{b p}{\zeta} g(t)} \exp \left\{-u \rho \frac{g(t) v(t)}{\xi}\right\} \\
& \times \mathbb{E}\left[v(T)^{u \frac{b \rho}{\zeta} g(T)} \exp \left\{u\left(\int_{t}^{T} B(s) v(s) d s+\int_{t}^{T} C(s) \frac{1}{v(s)} d s+\int_{t}^{T} D(s) \ln (v(s)) d s+\rho \frac{g(T) v(T)}{\xi}\right)\right\} \mid \mathcal{F}_{t}\right]
\end{aligned}
$$

where $A, B, C$ and $D$ are provided in the proof.

Proof. Proof of Lemma A1.

Let the generic process be:

$$
\begin{aligned}
& d Z(t)=\left[L(t)+h(t)\left(\sqrt{v(t)}+\frac{b}{\sqrt{v(t)}}\right)^{2}\right] d t+g(t)\left(\sqrt{v(t)}+\frac{b}{\sqrt{v(t)}}\right) d W_{t} \\
& d v(t)=\alpha(\theta-v(t)) d t+\xi \sqrt{v(t)} d B(t) \\
& \langle d B(t), d W(t)\rangle=\rho d t
\end{aligned}
$$

We want to find

$$
\mathbb{E}\left[e^{u Z(T)} \mid \mathcal{F}_{t}\right]=e^{u Z(t)} \Phi_{G G}\left(T, \phi ; L, h, g, \kappa, \theta, \xi, \rho, b, c, v_{t}, Z_{t}\right)
$$

Letting $\tilde{v}(t)=g(t) v(t)$ and $\hat{v}(t)=g(t) \ln (v(t))$, we have following:

$$
d \tilde{v}(t)=\alpha \theta g(t) d t+\left(g^{\prime}(t)-\alpha g(t)\right) v(t) d t+g(t) \xi \sqrt{v(t)} d B(t),
$$

and

$$
\begin{aligned}
d \hat{v}(t) & =\frac{g^{\prime}(t)}{g(t)} \hat{v}(t) d t+\frac{\partial \hat{v}(t)}{\partial \ln (v(t))} d \ln (v(t))+\frac{1}{2} \frac{\partial^{2} \hat{v}(t)}{\partial \ln (v(t))^{2}}<d \ln (v(t))> \\
& =\frac{g^{\prime}(t)}{g(t)} \hat{v}(t) d t+\frac{g(t) \xi}{\sqrt{v(t)}} d B(t)+g(t)\left(\frac{\alpha \theta}{v(t)}-\alpha\right) d t-g(t) \frac{\xi^{2}}{2 v(t)} d t
\end{aligned}
$$


From Equations (A9) and (A10), we solve for $\int_{t}^{T} g(s) \sqrt{v(s)} d B(s)$ and $\int_{t}^{T} \frac{g(s)}{\sqrt{v(s)}} d B(s)$ :

$$
\begin{gathered}
\int_{t}^{T} g(s) \sqrt{v(s)} d B(s)=\frac{g(T) v(T)-g(t) v(t)}{\zeta}-\frac{\alpha \theta}{\zeta} \int_{t}^{T} g(s) d s-\frac{1}{\zeta} \int_{t}^{T}\left(g^{\prime}(s)-\alpha g(s)\right) v(s) d s, \\
\int_{t}^{T} \frac{g(s)}{\sqrt{v(s)}} d B(s)=\frac{1}{\zeta} \ln \frac{v(T) g^{g(T)}}{v(t) g^{g(t)}}+\frac{\alpha}{\zeta} \int_{t}^{T} g(s) d s+\frac{1}{\zeta}\left(\frac{\tau^{2}}{2}-\alpha \theta\right) \int_{t}^{T} \frac{g(s)}{v(s)} d s-\frac{1}{\zeta} \int_{t}^{T} g^{\prime}(s) \ln (v(s)) d s .
\end{gathered}
$$

Split $W(t)$ into $B(t)$ and its orthogonal part $B(t)^{\perp}$ :

$$
\begin{aligned}
Z(T) & =Z(t)+\int_{t}^{T} L(s) d s+\int_{t}^{T} h(s)\left(\sqrt{v(s)}+\frac{b}{\sqrt{v(s)}}\right)^{2} d s \\
& +\int_{t}^{T} g(s)\left(\sqrt{v(s)}+\frac{b}{\sqrt{v(s)}}\right)\left(\rho d B(s)+\sqrt{1-\rho^{2}} d B(s)^{\perp}\right),
\end{aligned}
$$

then substitute Equation (A11) and (A12) to eliminate $d B(t) . Z(t)$ can be rewritten now as:

$$
\begin{aligned}
Z(T) & =Z(t)+\int_{t}^{T} L(s) d s+\int_{t}^{T} h(s)\left(v(s)+2 b+\frac{b^{2}}{v(s)}\right) d s+\sqrt{1-\rho^{2}} \int_{t}^{T} g(s)\left(a \sqrt{v(s)}+\frac{b}{\sqrt{v(s)}}\right) d B(s)^{\perp} \\
& +\rho \frac{g(T) v(T)-g(t) v(t)}{\xi}-\frac{\alpha \theta \rho}{\xi} \int_{t}^{T} g(s) d s-\frac{\rho}{\xi} \int_{t}^{T}\left(g^{\prime}(s)-\alpha g(s)\right) v(s) d s \\
& +\frac{b \rho}{\xi} \ln \frac{v(T)^{g(T)}}{v(t)^{g(t)}}+\frac{\alpha b \rho}{\xi} \int_{t}^{T} g(s) d s+\frac{b \rho}{\xi}\left(\frac{\xi^{2}}{2}-\alpha \theta\right) \int_{t}^{T} \frac{g(s)}{v(s)} d s-\frac{b \rho}{\xi} \int_{t}^{T} g^{\prime}(s) \ln (v(s)) d s
\end{aligned}
$$

Grouping conveniently, we obtain:

$$
\begin{aligned}
Z(T) & =Z(t)+\int_{t}^{T} \bar{A}(s) d s+\int_{t}^{T} \bar{B}(s) v(s) d s+\int_{t}^{T} \bar{C}(s) \frac{1}{v(s)} d s+\int_{t}^{T} D(s) \ln (v(s)) d s \\
& +\frac{b \rho}{\xi} \ln \frac{v(T)^{g(T)}}{v(t)^{g(t)}}+\rho \frac{g(T) v(T)-g(t) v(t)}{\xi}+\sqrt{1-\rho^{2}} \int_{t}^{T} g(s)\left(a \sqrt{v(s)}+\frac{b}{\sqrt{v(s)}}\right) d B(s)^{\perp}
\end{aligned}
$$

where

$$
\begin{aligned}
& \bar{A}(s)=L(s)+2 b h(s)+\left(\frac{\alpha b \rho}{\xi}-\frac{\alpha \theta \rho}{\xi}\right) g(s) \\
& \bar{B}(s)=h(s)-\frac{\rho}{\xi}\left(g^{\prime}(s)-\alpha g(s)\right) \\
& \bar{C}(s)=b^{2} h(s)+\frac{b \rho}{\xi}\left(\frac{\xi^{2}}{2}-\alpha \theta\right) g(s) \\
& D(s)=-\frac{b \rho}{\xi} g^{\prime}(s)
\end{aligned}
$$

Let $\left(\mathcal{G}_{t}\right)_{t \geq 0}$ denote the filtration generated by $v(t), t \geq 0$. Using iterated expectation and independence, we can write the conditional moment generating function of $Z(T)$ as:

$$
\begin{array}{r}
\mathbb{E}\left[e^{u Z(T)} \mid \mathcal{F}_{t}\right]=\mathbb{E}\left[\mathbb{E}\left[e^{u Z(T)} \mid \mathcal{F}_{t} \cup \mathcal{G}_{t}\right] \mid \mathcal{F}_{t}\right]=\exp \left\{u\left(Z(t)+\int_{t}^{T} A(s) d s\right)\right\} v(t)^{u \frac{b \rho}{\zeta} g(t)} \exp \left\{-u \rho \frac{g(t) v(t)}{\xi}\right\} \\
\times \mathbb{E}\left[\exp \left\{u\left(\int_{t}^{T} B(s) v(s) d s+\int_{t}^{T} C(s) \frac{1}{v(s)} d s+\int_{t}^{T} D(s) \ln (v(s)) d s+\frac{b \rho}{\xi} \ln v(T)^{g(T)}+\rho \frac{g(T) v(T)}{\xi}\right)\right\}\right. \\
\left.\times \mathbb{E}\left[\exp \left\{u \sqrt{1-\rho^{2}} \int_{t}^{T} g(s)\left(\sqrt{v(s)}+\frac{b}{\sqrt{v(s)}}\right) d B(s)^{\perp}\right\} \mid \mathcal{F}_{t} \bigcup \mathcal{G}_{t}\right] \mid \mathcal{F}_{t}\right]
\end{array}
$$


The inner expectation, conditioned on $\mathcal{F}_{t} \cup \mathcal{G}_{t}$, leads to a normal random variable with mean 0 and variance (Ito's Isometry) $u^{2}\left(1-\rho^{2}\right) \int_{t}^{T} g^{2}(s)\left(v(s)+\frac{b^{2}}{v(s)}+2 b\right) d s$. Putting all together:

$$
\begin{aligned}
& \mathbb{E}\left[e^{u Z(T)} \mid \mathcal{F}_{t}\right]=\exp \left\{u\left(Z(t)+\int_{t}^{T} A(s) d s\right)\right\} v(t)^{u \frac{b \rho}{\zeta} g(t)} \exp \left\{-u \rho \frac{g(t) v(t)}{\xi}\right\} \\
& \times \mathbb{E}\left[v(T)^{u \frac{b \rho}{\zeta} g(T)} \exp \left\{u\left(\int_{t}^{T} B(s) v(s) d s+\int_{t}^{T} C(s) \frac{1}{v(s)} d s+\int_{t}^{T} D(s) \ln (v(s)) d s+\rho \frac{g(T) v(T)}{\xi}\right)\right\} \mid \mathcal{F}_{t}\right]
\end{aligned}
$$

where

$$
\begin{aligned}
A(s) & =\bar{A}(s)+u\left(1-\rho^{2}\right) b g^{2}(s) \\
B(s) & =\bar{B}(s)+\frac{1}{2} u^{2}\left(1-\rho^{2}\right) g^{2}(s) \\
C(s) & =\bar{C}(s)+\frac{1}{2} u^{2}\left(1-\rho^{2}\right) b^{2} g^{2}(s)
\end{aligned}
$$

Proof. Proof of Corollary 1.

The proof starts similarly to Proposition 2. We start by defining new processes $d S_{i j, t}$ and $d S_{t}^{i}$ with $i, j=1,2, \ldots, n$ and $t \geq 0$.

$$
\begin{aligned}
d Z_{i}(t) & =\left[\left(\widetilde{c}_{i}-\frac{1}{2}\right)\left(\sqrt{\tilde{v}_{i}(t)}+\frac{\tilde{b}_{i}}{\sqrt{\tilde{v}_{i}(t)}}\right) d t+\left(\sqrt{\tilde{v}_{i}(t)}+\frac{\tilde{b}_{i}}{\sqrt{\tilde{v}_{i}(t)}}\right) d \widetilde{W}_{i}\right] \\
& +\sum_{j=1}^{n}\left[\frac{L_{i}}{n} d t+\left(c_{j}-\frac{1}{2}\right) a_{i j}^{2}\left(\sqrt{v_{j}(t)}+\frac{b_{j}}{\sqrt{v_{j}(t)}}\right)^{2} d t+a_{i j}\left(\sqrt{v_{j}(t)}+\frac{b_{j}}{\sqrt{v_{j}(t)}}\right) d W_{j}\right] \\
& =d S_{t}^{i}+\sum_{j=1}^{n} d S_{i j, t}
\end{aligned}
$$

By the dependence structure implied by the model, it follows that all $S$ are independent for a fix $i$, hence we can transform the characteristic function using the processes $S$ as follows:

$$
\Phi_{Z(t), v(t)}(T, \omega)=\prod_{j=1}^{n} E\left[\exp \left\{i \omega^{\prime}\left(S_{\cdot j, T}-S_{\cdot j, t}\right)\right\} \mid S_{t}, v(t)\right] \prod_{i=1}^{n} E\left[\exp \left\{i \omega_{i}\left(S_{T}^{i}-S_{t}^{i}\right)\right\} \mid S_{t}, v(t)\right]
$$

For each factor $j=1,2, \ldots, n$ we define $S_{j, t}^{*}=\omega^{\prime} S_{\cdot j, t}=\sum_{i=1}^{n} \omega_{i} S_{i j, t}$, the dynamics of $S_{j, t}^{*}$ can be expressed as

$$
\begin{aligned}
& d S_{j, t}^{*}=\omega^{\prime} d S_{\cdot j, t} \\
& =\left[L(\omega)+h_{j}(\omega)\left(\sqrt{v_{j}(t)}+\frac{b_{j}}{\sqrt{v_{j}(t)}}\right)^{2}\right] d t+g_{j}(\omega)\left(\sqrt{v_{j}(t)}+\frac{b_{k}}{\sqrt{v_{j}(t)}}\right) d W_{j, t}
\end{aligned}
$$

where $h_{j}(\omega)=\sum_{i=1}^{n} \omega_{i}\left(c_{i}-\frac{1}{2}\right) a_{i j}^{2}, L(\omega)=\sum_{i=1}^{n} \omega_{i} \frac{L_{i}}{n}$ and $g_{j}(\omega)=\sum_{i=1}^{n} \omega_{i} a_{i j}$. Next, we find the characteristic function for the increments of $S_{j, t}^{*}$ :

$$
E\left[\exp \left\{i \phi\left(S_{j, T}^{*}-S_{j, t}^{*}\right)\right\} \mid S_{j, t}^{*}, v_{j}(t)=v_{j, t}\right]=\Phi_{G}\left(T, \phi ; L, h_{j}, g_{j}, \kappa_{j}, \theta_{j}, \xi_{j}, \rho_{j}, b_{j}, c_{j}, v_{j, t}, S_{j, t}^{*}\right) .
$$

The generic function $\Phi_{G}$ is provided in the Appendix B. 
Similarly for the idiosyncratic factors $d S_{t}^{i}$ with $i=1,2, \ldots, n$ :

$$
d S_{t}^{* i}=\omega_{i} d S_{t}^{i}=h_{i}(\omega)\left(\sqrt{\tilde{v}_{i}(t)}+\frac{\tilde{b}_{i}}{\sqrt{\tilde{v}_{i}(t)}}\right)^{2} d t+g_{i}(\omega)\left(\sqrt{\tilde{v}_{i}(t)}+\frac{\tilde{b}_{i}}{\sqrt{\tilde{v}_{i}(t)}}\right) d \widetilde{W}_{i, t}
$$

where $h_{i}(\omega)=\omega_{i}\left(\widetilde{c}_{i}-\frac{1}{2}\right)$ and $g_{i}(\omega)=\omega_{i}$.

Combining all pieces together, we obtain:

$$
\begin{aligned}
& \Phi_{Z(t), v(t)}(T, \omega)=\prod_{j=1}^{n} E\left[\exp \left\{i \omega^{\prime}\left(S_{\cdot j, T}-S_{\cdot j, t}\right)\right\} \mid S_{t}, v(t)\right] \prod_{i=1}^{n} E\left[\exp \left\{i \omega_{i}\left(S_{T}^{i}-S_{t}^{i}\right)\right\} \mid S_{t}, v(t)\right] \\
& =\prod_{j=1}^{n} \Phi_{G}\left(T, \phi ; L, h_{j}, g_{j}, \kappa_{j}, \theta_{j}, \widetilde{\zeta}_{j}, \rho_{j}, b_{j}, c_{j}, v_{j, t}, S_{j, t}^{*}\right) \times \prod_{i=1}^{n} \Phi_{G}\left(T, 1 ; 0, h_{i}, g_{i}, \widetilde{\kappa}_{i}, \widetilde{\theta}_{i}, \widetilde{\zeta}_{i}, \widetilde{\rho}_{i}, \widetilde{b}_{i}, \widetilde{c}_{i}, \widetilde{v}_{i, t}, S_{t}^{* i}\right)
\end{aligned}
$$

Proof. Proof of Corollary 2.

The proof uses Corollary 1, where we express the joint c.f. as the product of one dimensional c.f.s of $4 / 2$ type.

$$
\begin{aligned}
& \Phi_{Z(t), v(t)}(T, \omega)=\prod_{j=1}^{n} E\left[\exp \left\{i \omega^{\prime}\left(S_{\cdot j, T}-S_{\cdot j, t}\right)\right\} \mid S_{t}, v(t)\right] \prod_{i=1}^{n} E\left[\exp \left\{i \omega_{i}\left(S_{T}^{i}-S_{t}^{i}\right)\right\} \mid S_{t}, v(t)\right] \\
& =\prod_{j=1}^{n} \Phi_{G}\left(T, \phi ; L, h_{j}, g_{j}, \kappa_{j}, \theta_{j}, \xi_{j}, \rho_{j}, b_{j}, c_{j}, v_{j, t}, S_{j, t}^{*}\right) \times \prod_{i=1}^{n} \Phi_{G}\left(T, 1 ; 0, h_{i}, g_{i}, \widetilde{\kappa}_{i}, \widetilde{\theta}_{i}, \widetilde{\xi}_{i}, \widetilde{\rho}_{i}, \widetilde{b}_{i}, \widetilde{c}_{i}, \widetilde{v}_{i, t}, S_{t}^{* i}\right)
\end{aligned}
$$

Hence, every one of these functions $\left(\Phi_{G}\left(T, \phi ; L, h, g, \kappa, \theta, \xi, \rho, b, c, v_{t}, Z_{t}\right)=\mathbb{E}\left[e^{u Z(T)} \mid \mathcal{F}_{t}\right]\right)$ capture the c.f. of a process of the type:

$$
\begin{aligned}
& d Z(t)=\left[L+h\left(\sqrt{v(t)}+\frac{b}{\sqrt{v(t)}}\right)^{2}\right] d t+g\left(\sqrt{v(t)}+\frac{b}{\sqrt{v(t)}}\right) d W_{t} \\
& d v(t)=\alpha(\theta-v(t)) d t+\xi \sqrt{v(t)} d B(t) \\
& \langle d B(t), d W(t)\rangle=\rho d t
\end{aligned}
$$

It is not difficult to realize therefore that the c.f. given $v(T)$ can be similarly computed for every one of those processes, hence one can infer:

$$
\begin{aligned}
\Phi_{Z(t), v(T)}(T, \omega) & =\prod_{j=1}^{n} \Phi_{G, T}\left(T, \phi ; L, h_{j}, g_{j}, \kappa_{j}, \theta_{j}, \widetilde{\xi}_{j}, \rho_{j}, b_{j}, c_{j}, v_{j, T}, S_{j, t}^{*}\right) \\
& \times \prod_{i=1}^{n} \Phi_{G, T}\left(T, 1 ; 0, h_{i}, g_{i}, \widetilde{\kappa}_{i}, \widetilde{\theta}_{i}, \widetilde{\xi}_{i}, \widetilde{\rho}_{i}, \widetilde{b}_{i}, \widetilde{c}_{i}, \widetilde{v}_{i, T}, S_{t}^{* i}\right)
\end{aligned}
$$

where $\Phi_{G, T}\left(T, \phi ; L, h, g, \kappa, \theta, \xi, \rho, b, c, v_{T}, Z_{t}\right)=\mathbb{E}\left[e^{u Z(T)} \mid \mathcal{F}_{t} \cup v(T)\right]$ is provided next in Appendix B. 


\section{Appendix B. Helpful Results}

Given the $4 / 2$ process, the following c.f. are used in this paper:

$$
\begin{aligned}
& d Z(t)=\left[L+h\left(\sqrt{v(t)}+\frac{b}{\sqrt{v(t)}}\right)^{2}\right] d t+g\left(\sqrt{v(t)}+\frac{b}{\sqrt{v(t)}}\right) d W_{t} \\
& d v(t)=\alpha(\theta-v(t)) d t+\xi \sqrt{v(t)} d B(t) \\
& \langle d B(t), d W(t)\rangle=\rho d t
\end{aligned}
$$

$$
\begin{aligned}
& \Phi_{G}\left(T, u ; L, h, g, \alpha, \theta, \xi, \rho, b, c, v_{t}, Z_{t}\right)=\mathbb{E}\left[e^{u Z(T)} \mid \mathcal{F}_{t}\right] \\
& =\exp \left\{u Z(t)+\frac{\alpha^{2} \theta}{\xi^{2}}(T-t)+u\left(r+2\left(h-\frac{1}{2}\right) g^{2} b-\frac{g \rho \alpha \theta}{\xi}+\frac{g b \rho \alpha}{\xi}\right)(T-t)+u^{2}\left(1-\rho^{2}\right) g^{2} b(T-t)\right\} \\
& \times\left(\frac{\sqrt{A_{u}}}{\xi^{2} \sinh \left(\frac{\sqrt{A_{u}}}{2} t\right)}\right)^{m_{u}+1} v(t)^{\frac{1}{2}+\frac{m_{u}}{2}-\frac{\alpha \theta}{\xi^{2}}-\frac{u g b \rho}{\xi}}\left(K_{u}(T)-\frac{u g \rho}{\xi}\right)^{-\left(\frac{1}{2}+\frac{m_{u}}{2}+\frac{\alpha \theta}{\xi^{2}}+\frac{u g b \rho}{\zeta}\right)} \\
& \times \exp \left\{\frac{v(t)}{\xi^{2}}\left(-\sqrt{A_{u}} \operatorname{coth}\left(\frac{\sqrt{A_{u}}(T-t)}{2}\right)+\alpha-u g \rho \xi\right)\right\} \frac{\Gamma\left(\frac{1}{2}+\frac{m_{u}}{2}+\frac{\alpha \theta}{\xi^{2}}+\frac{u g b \rho}{\xi}\right)}{\Gamma\left(m_{u}+1\right)} \\
& \times F_{1} F_{1}\left(\frac{1}{2}+\frac{m_{u}}{2}+\frac{\alpha \theta}{\xi^{2}}+\frac{u g b \rho}{\xi}, m_{u}+1, \frac{A_{u} v(t)}{\xi^{4}\left(K_{u}(T)-\frac{u g \rho}{\zeta}\right) \sinh ^{2}\left(\frac{\sqrt{A_{u}}(T-t)}{2}\right)}\right),
\end{aligned}
$$

with

$$
\begin{aligned}
A_{u} & =\alpha^{2}-2 \xi^{2}\left(u\left(\frac{g \rho \alpha}{\xi}+\left(h-\frac{1}{2}\right) g^{2}\right)+\frac{1}{2} u^{2}\left(1-\rho^{2}\right) g^{2}\right), \\
m_{u} & =\frac{2}{\xi^{2}} \sqrt{\left(\alpha \theta-\frac{\xi^{2}}{2}\right)^{2}-2 \xi^{2}\left(u\left(\frac{g b \rho}{\xi}\left(\frac{\xi^{2}}{2}-\alpha \theta\right)+\left(h-\frac{1}{2}\right) g^{2} b^{2}\right)+\frac{1}{2} u^{2}\left(1-\rho^{2}\right) g^{2} b^{2}\right),} \\
K_{u}(T) & =\frac{1}{\xi^{2}}\left(\sqrt{A_{u}} \operatorname{coth}\left(\frac{\sqrt{A_{u}}(T-t)}{2}\right)+\alpha\right)
\end{aligned}
$$

$$
\begin{aligned}
& \Phi_{G, T}\left(T, u ; L, h, g, \kappa, \theta, \xi, \rho, b, c, v_{t}, Z_{t}\right)=\mathbb{E}\left[e^{u Z(T)} \mid \mathcal{F}_{t} \cup v(T)\right] \\
& =\exp \left\{u Z(t)+u\left(r+2\left(h-\frac{1}{2}\right) g^{2} b-\frac{a \rho \alpha \theta}{\xi}+\frac{b \rho \alpha}{\xi}\right)(T-t)+u^{2}\left(1-\rho^{2}\right) g^{2} b(T-t)\right\} \\
& \times \exp \left\{\frac{u g \rho}{\xi}(v(T)-v(t))+\frac{u g b \rho}{\xi} \log \frac{v(T)}{v(t)}\right\} \\
& \times \frac{\sqrt{A_{u}} \sinh \left(\frac{\alpha(T-t)}{2}\right)}{\alpha \sinh \left(\frac{\sqrt{A_{u}}(T-t)}{2}\right)} \exp \left(\frac{v(T)+v(t)}{\xi^{2}}\left(\alpha \operatorname{coth}\left(\frac{\alpha(T-t)}{2}\right)-\sqrt{A_{u}} \operatorname{coth}\left(\frac{\sqrt{A_{u}}(T-t)}{2}\right)\right)\right) \\
& \times \frac{I_{\bar{\xi}^{2}} \sqrt{\left(\alpha \theta-\frac{\xi^{2}}{2}\right)^{2}+2 \tilde{\xi}^{2} B_{u}}\left(\frac{2 \sqrt{A_{u} v(T) v(t)}}{\tilde{\xi}^{2} \sinh \left(\frac{\sqrt{A_{u}(T-t)}}{2}\right)}\right)}{I_{\frac{2 \alpha \theta}{\xi^{2}}-1}\left(\frac{2 \alpha \sqrt{v(T) v(t)}}{\tilde{\xi}^{2} \sinh \left(\frac{\alpha(T-t)}{2}\right)}\right)},
\end{aligned}
$$

with

$$
B_{u}=u\left(\frac{g b \rho}{\xi}\left(\frac{\xi^{2}}{2}-\alpha \theta\right)+\left(h-\frac{1}{2}\right) g^{2} b^{2}\right)+\frac{1}{2} u^{2}\left(1-\rho^{2}\right) g^{2} b^{2}
$$




\section{References}

Baldeaux Jan, Martino Grasselli, and Eckhard Platen. 2015. Pricing Currency Derivatives Under the Benchmark Approach. Journal of Banking \& Finance 53: 34-48.

Carr, Peter, and Dilip B. Madan. 1999. Option Valuation using the FFT. Journal of Computational Finance 2: 61-73. [CrossRef]

Caldana, Ruggero, and Gianluca Fusai. 2013. A general closed-form spread option pricing formula. Journal of Banking and Finance 37: 4893-906. [CrossRef]

Cheng, Peng, and Olivier Scaillet. 2007. Linear-quadratic jump-diffusion modelling. Mathematical Finance 17: 575-98. [CrossRef]

Chiarella, Carl, Boda Kang, Christina Sklibosios Nikitopoulos, and Thuy Tô. 2013. Humps in the volatility structure of the crude oil futures market: New evidence. Energy Economics 40: 989-1000. [CrossRef]

Christoffersen, Peter, Steven Heston, and Kris Jacobs. 2009. The shape and term structure of the index option smirk: Why multifactor stochastic volatility models work so well. Management Science. 55: 1914-32. [CrossRef]

De Col, Alvise, Alessandro Gnoatto, and Martino Grasselli. 2013. Smiles all around: FX joint calibration in a multi-Heston model. Journal of Banking \& Finance 37: 3799-818.

Da Fonseca, José, Martino Grasselli, and Claudio Tebaldi. 2007. Option Pricing when Correlations are Stochastic: An Analytical Framework. Review of Derivatives Research 10: 151-80. [CrossRef]

DeMiguel, Victor, Lorenzo Garlappi, and Raman Uppal. 2007. Optimal versus naive diversification: How inefficient is the $1 / \mathrm{N}$ portfolio strategy? The Review of Financial Studies 22: 1915-53. [CrossRef]

Diebold, Francis X., and Marc Nerlove. 1989. The dynamics of exchange rate volatility: A multivariate latent factor ARCH model. Journal of Applied Econometrics 4: 1-21. [CrossRef]

Escobar, Marcos. 2018. A Stochastic Volatility Factor Model of Heston type. Statistical Properties and Estimation. Stochastics 90: 172-99. [CrossRef]

Escobar, Marcos, and Christoph Gschnaidtner. 2016. Parameters recovery via calibration in the Heston model: A comprehensive review. Wilmott 2016: 60-81. [CrossRef]

Escobar, Marcos, and Zhenxian Gong. 2019. The mean-reverting 4/2 stochastic volatility model: Properties and Financial Applications. submitted.

Engle, Robert. 2002. Dynamic Conditional Correlation: A Simple Class of Multivariate Generalized Autoregressive Conditional Heteroskedasticity Models. Journal of Business \& Economic Statistics 20: 339-50.

Fusai, Gianluca, Marina Marena, and Andrea Roncoroni. 2018. Analytical pricing of discretely monitored Asianstyle options: Theory and application to commodity markets. Journal of Banking and Finance 32: 2033-45. [CrossRef]

Gourieroux, Christian. 2006. Continuous Time Wishart Process for Stochastic Risk. Econometric Reviews 25: 1-41. [CrossRef]

Grasselli, Martino. 2017. The 4/2 stochastic volatility model: A unified approach for the Heston and the 3/2 model. Mathematical Finance 27: 1013-34. [CrossRef]

Gnoatto, Alessandro, Martino Grasselli, and Eckhard Platenn. 2018. A penny saved is a penny earned: Less expensive zero coupon bonds. arXiv, arXiv:1608.04683.

Heston, Steven L. 1993. A Closed-Form Solution for Options with Stochastic Volatility with Applications to Bonds and Currency Options. Review of Financial Studies 6: 327-43. [CrossRef]

Medvedev Alexey, and Olivier Scaillet. 2007. Approximation and calibration of short-term implied volatilities under jump-diffusion stochastic volatility. Review of Financial Studies 20: 427-59. [CrossRef]

Muhle-Karbe, Johannes, Oliver Pfaffel, and Robert Stelzer. 2012. Option pricing in multivariate stochastic volatility models of OU type. SIAM Journal on Financial Mathematics 3: 66-94. [CrossRef]

Platen, Eckhard. 1997. A Non-Linear Stochastic Volatility Model. Financial Mathematics Research Report No. FMRR 005-97. Canberra: Center for Financial Mathematics, Australian National University.

Platen, Eckhard, and David Cochran Heath. 2010. A Benchmark Approach to Quantitative Finance. Berlin and Heidelberg: Springer. 
Schneider Lorenz, and Bertrand Tavin. 2018. From the Samuelson volatility effect to a Samuelson correlation effect: An analysis of crude oil calendar spread options. Journal of Banking and Finance 95: 185-202. [CrossRef]

Schwartz, Eduardo S. 1997. The stochastic behavior of commodity prices: Implications for valuation and hedging. The Journal of Finance 52: 923-73. [CrossRef]

(C) 2019 by the authors. Licensee MDPI, Basel, Switzerland. This article is an open access article distributed under the terms and conditions of the Creative Commons Attribution (CC BY) license (http:/ / creativecommons.org/licenses/by/4.0/). 